\title{
Abhandlungen
}

Michael Eßig und Alexander Batran

\section{Konzeptionelle Grundlagen des Public Supply Chain Management}

Multi-Ebenen-Netzwerk; Netzwerke; öffentliche Beschaffung; Public Supply Chain; Supply Chain Management; Wertschöpfung

Ausgangspunkt der vorliegenden Arbeit sind Entwicklungen sowohl des privatwirtschaftlichen Supply Chain Management, als auch Veränderungen im öffentlichen Sektor hin zu einer stärker marktlich geprägten Steuerung. Die Autoren greifen auf Konzeptbausteine des privatwirtschaftlichen Supply Chain Mangement zurück und entwickeln vor dem Hintergrund der Besonderheiten des öffentlichen Sektors konzeptionelle Grundlagen für ein Public Supply Chain Management. Der Netzwerkgedanke spielt hierbei eine weit größere Rolle, indem Ebenen der Politik, Administration und Privatwirtschaft simultan betrachtet werden müssen.

\section{Vorbemerkungen}

1. Zur Notwendigkeit eines Public Supply Chain Management

Man mag darüber diskutieren, ob die Ökonomisierung vieler gesellschaftlicher Bereiche zu weit führt oder erst am Anfang steht. ${ }^{1}$ Faktisch ist gesellschaftlich-öffentliches Handeln heute weitgehend fiskalisch und damit durch den Umgang mit Knappheit dominiert. Mit dem Ansatz des Public Supply Chain Management (Public SCM) soll der Versuch unternommen werden, einerseits eine bewusst betriebswirtschaftlich-ökonomische Perspektive für die Steuerung öffentlicher Wertschöpfung einzusetzen und andererseits nicht die damit häufig verbundene einseitig auf Effizienzsteigerung (sprich Kostenreduzierung) ausgerichtete Zieldimension zu verbinden.

In einem ersten Schritt werden dazu Impulse verschiedenster Forschungsgebiete wie öffentliche Beschaffung, Public Private Partnerships, New Public Management und schließlich (privatwirtschaftliches) Supply Chain Management aufgegriffen (Abschnitte 1. bis 4., Kapitel I). Damit wird ein Bezugsrahmen im Sinne einer Strukturierung vorhandenen Wissens hergestellt (vgl. Abbildung 1). ${ }^{2}$ Dieser dient als Basis für die Entwicklung einer

1 Vgl. Arnold (2004), S. 143.

2 Vgl. Rößl (1990), S. 99 ff. 
Public SCM-Konzeption ${ }^{3}$, welche wiederum in einem zweiten Schritt die Definition und Abgrenzung des Analyseobjektes Public Supply Chain gegenüber der „klassischen“ privatwirtschaftlichen Supply Chain (Abschnitt 1., Kapitel II) und in einem dritten Schritt die eigentlichen Konzeptmerkmale (Abschnitt 2., Kapitel II) beinhaltet. Abbildung 1 fasst diesen Bezugsrahmen zusammen.

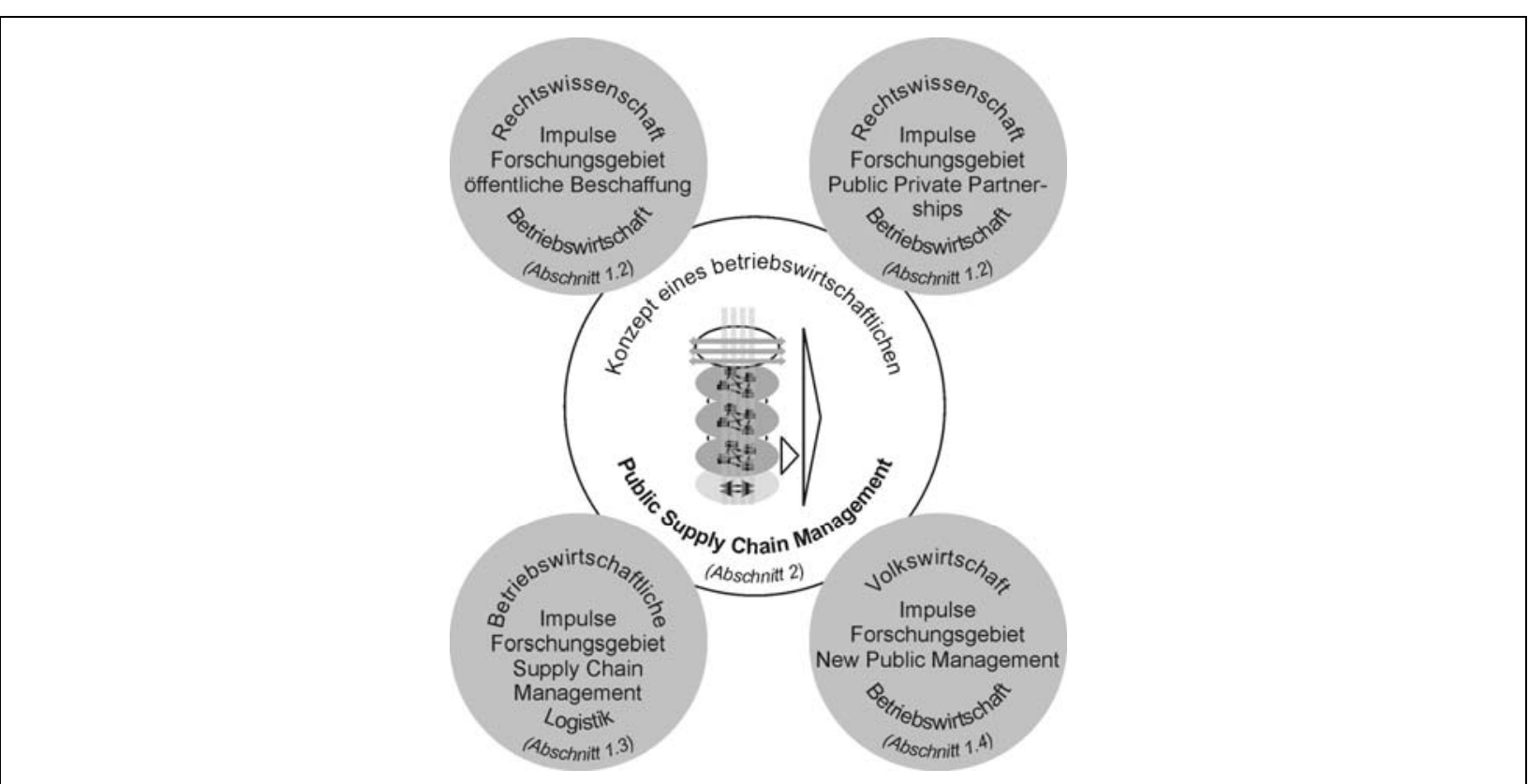

Abb. 1: Schritte der Konzeptentwicklung

Quelle: $\quad$ Rössl (1990), S. 99

2. Impulse der Forschungsgebiete öffentliche Beschaffung und Public Private Partnerships

Die öffentliche Beschaffung (Public Procurement) unterliegt den normativen Regelungen des Vergaberechts. ${ }^{4}$ Bereits der Begriff Vergabe macht deutlich, dass sich dahinter bis heute häufig noch das Verständnis eines hoheitlichen Aktes verbirgt, bei dem die öffentliche Hand privaten Unternehmen das Recht gewährt, sie beliefern zu dürfen. Die Diskussion um eine Reform des Vergaberechts ist bislang weitgehend durch juristische, wettbewerbspolitische und investitionspolitische Überlegungen determiniert. ${ }^{5}$

Tatsächlich stellen die juristische und makroökonomische Bewertung des Vergaberechts wesentliche, keineswegs aber die einzigen Dimensionen zur Weiterentwicklung der öffentlichen Beschaffung dar. Begleitet wird die Diskussion durch die zunehmende Privatisierung öffentlicher Leistungen oder die kooperative Zusammenarbeit mit privaten Anbietern. Insbesondere dann, wenn Leistungen auch von privaten Dritten erstellt werden können, sind Leistungen öffentlicher Institutionen im direkten Leistungsvergleich, auch

3 Vgl. zum Begriff der Konzeption Stölzle (1999), S. 145 f.

4 Vgl. o.V. (2001).

5 Vgl. Gründbuch der Kommission der europäischen Gemeinschaften (2004) sowie Arbeitsentwurf des Bundesministerium für Wirtschaft und Arbeit (2004). 
ohne vergleichende Marktpreise, mit einem betriebswirtschaftlichen Fokus zu analysieren. ${ }^{6}$ Primär sind öffentliche Beschaffungsaktivitäten somit die Schnittstelle zur Privatwirtschaft.

Das Vergaberecht ist mit seinem Primat einer an größtmöglicher Wirtschaftlichkeit orientierten Vergabeentscheidung nur vermeintlich an einer kaufmännisch optimalen Lösung orientiert. ${ }^{7}$ Tatsächlich wird das Wettbewerbsprinzip ${ }^{8}$ postuliert und konkret in einer Präferenz der „öffentlichen Ausschreibung“ (im EU-Duktus „offenes Verfahren“) bzw. ein Competitive Tendering-Verfahren (vgl. Abbildung 2) als Regelverfahren für den Einkauf der öffentlichen Hand umgesetzt. ${ }^{9}$ Leistungen werden im Competitive TenderingVerfahren „nach öffentlicher Aufforderung einer unbeschränkten Zahl von Unternehmen zur Einreichung von Angeboten “"10 vergeben. Kraft europarechtlicher Normierung, ${ }^{11}$ aber auch nach den $\S \S 97 \mathrm{ff}$. GWB (nebst Vergabeverordnung und Verdingungsordnungen) sind für das Vergabeverfahren die Grundsätze der Diskriminierungsfreiheit, der Transparenz und der Wettbewerbsoffenheit uneingeschränkt maßgeblich. Aus mikroökonomischbetriebswirtschaftlicher Perspektive gilt der „reine“ Lieferantenwettbewerb keinesfalls als Optimum. Zur Gewährleistung der Versorgung mit knappen Gütern sind Lieferantenpartnerschaften ein adäquates Mittel. Das Ziel ist, für den eigenen Erfolg kritische Lieferanten langfristig zu binden. ${ }^{12}$ Zudem ziehen Ausschreibungsverfahren häufig exorbitante Transaktionskosten nach sich, ohne im Ergebnis den leistungsfähigsten Anbieter auszuwählen. ${ }^{13}$ Privatunternehmen nehmen eine Beschränkung des Lieferantenwettbewerbs bei ihren Beschaffungsentscheidungen schon lange - und sehr bewusst - in Kauf. ${ }^{14}$ Mühlenkamp versucht als Interpretation das ökonomische Minimal- bzw. Maximalprinzip ${ }^{15}$ auf die - auch in Haushaltordnungen (BHO, LHO) und Haushaltgrundsätzgesetz - geforderten Prinzipien der Wirtschaftlichkeit und der Sparsamkeit zu übertragen. ${ }^{16}$ Bei alleiniger Betrachtung von Produktionskosten mag eine Übereinstimmung von Wirtschaftlichkeit mit dem Maximalprinzip und Sparsamkeit mit dem Minimalprinzip nachvollziehbar sein. Werden jedoch Transaktionskosten mit in das Kalkül miteinbezogen, so ist die Vereinbarkeit von Wettbewerb mit Wirtschaftlichkeit ungleich schwerer, beziehen sich dann ökonomische Prinzipien auf die Summe aus Produktions- und Transaktionskosten. Das Primat der öffentlichen Ausschreibung bleibt selbst dann bestehen, wenn die konkrete

6 Vgl. Budäus (1988), S. 208 ff., Borins/Grüning (1998), S. 35, ,... Bieterwettbewerb von privaten und öffentlichen Anbietern ...."

7 Vgl. § 25 Nr. 3 VOL/A, sinngemäß auch in $\S 25$ Nr. 3 (3) VOB/A und § 16 VOF, „Der Zuschlag ist auf das unter Berücksichtigung aller Umstände wirtschaftlichste Angebot zu erteilen. Der niedrigste Angebotspreis allein ist nicht entscheidend.“

8 Vgl. § 2 Nr. 1 Satz 2 VOB/A, „Der Wettbewerb soll die Regel sein“, § 2 Nr. 1 (1) VOL/A, „Leistungen sind in der Regel im Wettbewerb zu vergeben.“

$9 \mathrm{Vgl} . \S 3 \mathrm{Nr} .2 \mathrm{VOB} / \mathrm{A}, \S 3 \mathrm{Nr} .2 \mathrm{VOL} / \mathrm{A}$.

$10 \S 3 \mathrm{Nr}$. (1) VOB/A und VOL/A.

11 Vgl. Vergaberichtlinien, EuGH-Rechtsprechung.

12 Vgl. Lambert/Emmelhainz/Gardner (1996), S. 1.

13 Vgl. BME/Booz, Allen \& Hamilton (2000), S. 29 und S. 31.

14 Vgl. Owens Swift/Coe (1994), S. 172 ff.

15 Vgl. Schweitzer (2000), S. 63.

16 Vgl. Mühlenkamp (2003), S. 53 und S. 56 ff. 
Beschaffungssituationen zur Transaktionskostensenkung beziehungsorientierte Zulieferer-Abnehmer-Partnerschaften erfordert. ${ }^{17}$

Intensive Beziehungen zwischen Abnehmer und Lieferant entstehen durch die vermehrte Verlagerung von know-how-intensiven Wertschöpfungsanteilen an Systemzulieferer. Diese liefern nicht mehr klassische Zulieferteile, sondern ganze Leistungsbündel, bestehend aus Sach- und Dienstleistungen. ${ }^{18}$ Beispiel hierfür ist die Beschaffung schlüsselfertiger Bürokonzepte inklusive des kompletten Facility Management, d.h. Bau, Betrieb, Wartung anstatt einer separaten Ausschreibung einzelner Baulose und dem anschließenden Eigenbetrieb. ${ }^{19}$ Die fallweise Bevorzugung von marktlichen und hybriden Steuerungsmechanismen ist im Public Procurement ungleich schwerer, da neben rein (mikro-) ökonomischen Zielen weitere makroökonomische, regulatorische sowie soziale Zielperspektiven eingehalten werden müssen. Erridge und McIlroy ordnen hybride Ausprägungen in Form von Partnerschaften und längerfristigen Verträgen einem rein „kommerziellen“ (ökonomischen) Beschaffungstyp zu. Dagegen führt die Anwendung von EURichtlinien sowie Vergaberechtsvorschriften zu einem regulatorischen (Transparenz, Wettbewerb) und einem sozialen Typ (öffentliches Interesses, Umwelt), der einen langfristigen Beziehungshorizont nicht vorsieht. ${ }^{20}$ Diese Überlegungen machen deutlich, dass Ziele des privatwirtschaftlichen Supply Chain Management (von uns in der Folge zur Abgrenzung als Private SCM bezeichnet) durch rechtliche Vorgaben nicht im gleichen Maße bzw. nur auf Umwegen zu erreichen sind. ${ }^{21}$ Dennoch unterliegen sowohl der private als auch der öffentliche Sektor dem Primat der Wirtschaftlichkeit. Einerseits aufgrund eines tatsächlich kaufmännischen Kalküls (,Supply Chain Value ${ }^{\text {‘22 }}$ ), andererseits aufgrund gesetzlicher Forderungen. Diese Gemeinsamkeit stellt eine erste Grundlage für eine betriebswirtschaftliche Analyse dar. ${ }^{23}$

Hinzu kommt, dass bei Verlagerung auf Zulieferer nicht nur ex ante Vertragsabschluss hohe Such- und Informationskosten anfallen, auch die ex post-Kontrolle der Qualität von Zulieferleistungen ist bei beratungsintensiven Gütern mit Vertrauenseigenschaften nur zu prohibitiv hohen Kosten möglich. ${ }^{24}$ Will man eine Wissens- bzw. Know-how-Leistung vollständig prüfen, muss man über den gleichen Wissensstand wie der Lieferant verfügen - und in diesem Fall benötigt man seine Leistung nicht mehr. ${ }^{25}$ Andererseits kann heute keine Institution mehr Wissen in allen Bereichen vorhalten, sondern muss sich auf einen

17 Vgl. § 3, Nr. 4, VOL/A, § 3, Nr. 4, VOB/A, mit Ausnahme § 5 VOF, freihändige Vergabe nur unter bestimmten Voraussetzungen.

18 Vgl. Engelhardt/Kleinaltenkamp/Reckenfelderbäumer (1993).

19 Vgl. Präuer (2004).

20 Vgl. Erridge/McIlroy (2003), S. 548.

21 Vgl. Erridge/McIlroy (2003), S. 548.

22 Vgl. Neher (2003), S. 32 entwickelt ein Konzept zur Bestimmung des Supply Chain Value. Dabei spielt sowohl die Kundenperspektive („Customer perceived Value“) als auch die Lieferantenperspektive eine wichtige Rolle (,Supplier perceived Value“).

23 Vgl. Eichhorn (2001), S. 410.

24 Vgl. Adler (1996); Darby/Karni (1973); Nelson (1970); Weiber/Adler (1995).

25 Vgl. Göbel (2004), S. 19. Die Informationsasymmetrie ist bei Vetrauensgütern nicht ohne Weiteres abbaubar. Bspw. kann eine Stadt ein Risikogutachten nur dadurch prüfen, indem sie dieselben Information selbst erhebt. Dadurch wird aber die Fremdvergabe ad absurdum geführt. 
Kern an Kompetenzen konzentrieren. ${ }^{26}$ Andernfalls werden ökonomische Vorteile der Spezialisierung nicht realisiert. Nicht nur die Prüfung einer Leistung ex post kann Probleme bereiten, häufig sind Leistungen ex ante nur sehr schwer in konkreten Lösungen spezifizierbar. Im Rahmen eines Konzeptwettbewerbs sollen daher Lieferanten kreative Vorschläge zur Lösung machen. ${ }^{27}$ Als Konsequenz setzen Unternehmen heute nicht mehr ausschließlich auf marktlich-kurzfristige bzw. wettbewerbsorientierte Beziehungen zu ihren präferierten Systemlieferanten. Statt dessen entwickeln sie Partnerschaftsprogramme, die eine Auswahl von Lieferanten über Konzeptwettbewerbe und eine langfristige Lieferbindung (,life of product supply ${ }^{628}$ ) vorsieht. Dies führt bspw. zum Aufbau von Vertrauen als transaktionskostensenkende Maßnahme. ${ }^{29}$ War die frühe Einbeziehung von bzw. der Dialog mit Lieferanten bislang durch die vorgeschriebene Präferenz der öffentlichen Ausschreibung untersagt, werden aus vergaberechtlicher Sicht „neue“ Formen der Zusammenarbeit mit Zulieferern bei wissensintensiven Zulieferleistungen bereits erstmalig mit der Vergabeform des „Wettbewerblichen Dialogs“ der neuen EU-Richtlinie vom März 2004 berücksichtigt. ${ }^{30}$ Damit wird zumindest bei der Partnersuche der Beziehungsgedanke vergaberechtskonform. Des Weiteren tritt zu den bisherigen Verfahren die Möglichkeit zum Abschluss von zeitlich limitierten Rahmenvereinbarungen hinzu, womit Lieferabrufe einer gleichbleibenden Leistung vereinfacht werden. ${ }^{31}$ Klauseln bei der Vergabeentscheidung zur Bevorzugung ,bekannter und bewährter“"Bieter dienen der Vergabestelle, sich eine wiederholende umfangreiche Prüfung immer wieder mitbietender Unternehmen zu sparen (Klausel als Indiz für Zuverlässigkeit und Leistungsfähigkeit). Im Einzelfall kann es aber zu einer rechtswidrigen Bevorzugung eines Lieferanten (bspw. des In-Supplier) führen, so dass selbst durch die Aufnahme dieser Klausel eine langfristige Beziehung vergaberechtswidrig ist. ${ }^{32}$ Dennoch ist die Reform des Vergaberechts auf der Basis der EU-Richtlinien 2004/18/EG und 2004/17/EG als Tendenz zu einem strategisch orientierten Ansatz der öffentlichen Beschaffung zu bewerten. Das Dilemma der öffentlichen Beschaffung resultiert einerseits aus der Forderung nach mehr Wettbewerb zur effizienten Allokation von Ressourcen sowie andererseits den Erfolgen der Privatwirtschaft durch strategisches Supplier Relationship Management, also dem expliziten und fallweisen Ausschluss von Wettbewerb. ${ }^{33}$ Folgt man der Argumentation der Public Choice Theorie fehlen Institutionen des öffentlichen Sektors Steuerungs- und Anreizmechanismen, um der individuellen Nutzen- und Wohlstandsmaximierung der handelnden Akteure geeignet entgegenzuwirken. ${ }^{34}$ Theoretische Lösungsansätze liefern sowohl die Prinzipal-

26 Vgl. Prahalad/Hamel (1990).

27 Vgl. Backhaus (2003), S. 757 f., Bozdogan u.a. (1998), S. 164, Early Supplier Integration.

28 Harding/Harding (1991), S. 29 f.

29 Vgl. Bartelt (2002), S. $103 \mathrm{f}$.

30 Vgl. § 3, Nr. 1 (4) VOL/A, § 3, Nr. 2, VOB/A, Artikel 29, Richtlinie 2004/18/EG.

31 Artikel 32, Richtlinie 2004/18/EG ,... nach den Bedingungen der Rahmenvereinbarung ohne erneuten Aufruf zum Wettbewerb ...“ und Artikel 31, Richtlinie 2004/18/EG ,... die Laufzeit dieser Aufträge sowie der Daueraufträge darf in der Regel drei Jahre nicht überschreiten.“

32 Vgl. Riese (1998), S. 216, darauf aufbauend Fante (2004), S. 39 ff. und S. 313, § 20 GWB, Ungleichbehandlung von Bietern.

33 Vgl. Erridge/McIlroy (2003), S. 550; vgl. zu Wertschöpfungspartnerschaften Arnold (1993); Bogaschewsky (1994); Wildemann (2005), S. 505-507.

34 Vgl. Harms/Reichard (2003), S. 14 und Löffler (2003), S. 21. 
Agent-Theorie als auch die Transaktionskostentheorie, die im Rahmen einer PublicPrivate-Partnership Diskussion eine besondere Rolle einnimmt (vgl. Abbildung 2). ${ }^{35}$ Aus makroökonomischer Sicht bleibt es unklar, ob mit den genannten Grundsätzen, insbesondere der Orientierung am Wettbewerbsprinzip, neben einem mikroökonomisch, betriebswirtschaftlich (aus Sicht des einzelnen öffentlichen Auftraggebers) „optimalem“ Wettbewerb, ein (aus Sicht der Allgemeinheit als Ganzes) volkswirtschaftlich ,optimaler" Wettbewerb erreicht wird. Die Präferenz einer losweisen Ausschreibung ebenso wie das Diskriminierungsverbot lassen vermuten, dass mittelstandsorientierte, polypolistische Strukturen als wettbewerbsintensivst angesehen werden. ${ }^{36}$ Ohne hier auf Details der volkswirtschaftlichen Wettbewerbstheorie einzugehen, sei darauf verwiesen, dass im Gegensatz dazu auch weite Oligopole als wettbewerbsoptimal gelten - mithin ein gesamtwirtschaftliches Optimum durch das Vergaberecht nicht zwingend erreicht wird. ${ }^{37}$

Aus Sicht der betriebswirtschaftlichen Beschaffungsforschung wird der beziehungsorientierte Einkauf auch als Supply Management bezeichnet. ${ }^{38}$ Damit ist bereits der Führungsanspruch des Einkaufs verbunden, Wertschöpfungsprozesse und -strukturen in Zusammenarbeit mit Lieferanten neu zu gestalten. Public Supply Management steht für ein Führungskonzept im Einkauf aus Sicht öffentlicher Auftraggeber (vgl. Abbildung 2).

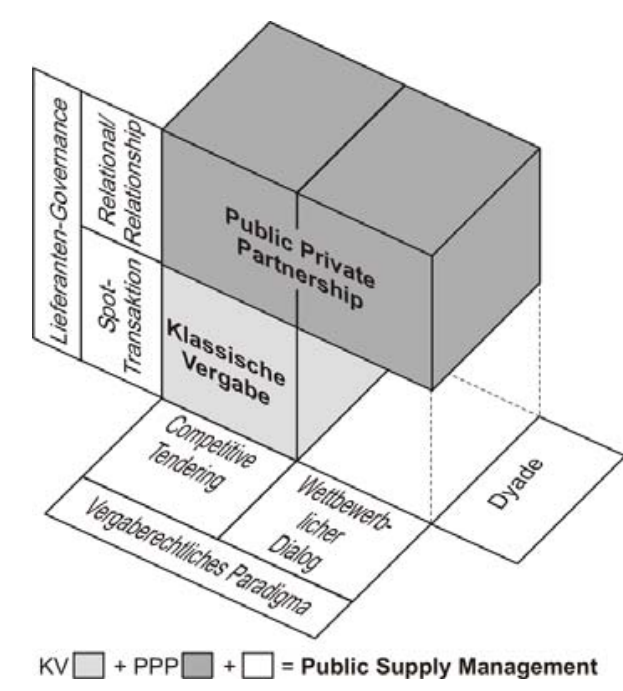

Abb. 2: Klassische Vergabe, Public Private Partnerships und Public Supply Management

Quelle: $\quad$ Eigene Darstellung

Mit Public Private Partnerships (PPP) existieren bereits heute intensive Formen der Zusammenarbeit zwischen öffentlichen Institutionen und privatwirtschaftlichen Lieferanten zur Verfolgung gemeinsamer Ziele. ${ }^{39}$ PPPs werden sowohl empirisch als auch wissen-

35 Vgl. Zafirowski (2001), S. 674.

36 Vgl. § 2, Nr. 2, § 5, Nr.1 VOL/A, § 2, Nr. 2 sowie $\S 4$, Nr. 2 VOB, § 4 (2), § 20 und eingeschränkt $§ 4$ (5) VOF.

37 Vgl. Kantzenbach (1967), S. 49 bzw. beispielhaft die Diskussion bei Mantzavinos (1994), S. 65 ff.; Oberender (1995), S. $36 \mathrm{ff}$.

38 Vgl. Arnold (1997), Bogaschewsky (2003); Burt/Dobler/Starling (2003), Eßig (2005), S. 8.

39 Vgl. Eichhorn (1995), S. 174. 
schaftlich breit rezipiert. Faktisch stellen PPPs - wie rein privatwirtschaftliche Abnehmer-Zulieferer-Kooperationen - hybrid, d.h. gemischt marktlich-hierarchisch gesteuerte Institutionen dar und führen zu einer Neuverteilung der Aufgaben in der öffentlichen Wertschöpfungskette. ${ }^{40}$ PPPs erstrecken sich sowohl auf rein vertragliche Formen ${ }^{41}$, als auch auf die Gründung eines öffentlich-privaten Gemeinschaftsunternehmens. ${ }^{42}$ Die ökonomische Begründung zur Anwendung von PPPs liefern einerseits Überlegungen zur strategischen Bedeutung des Outsourcing-Objekts und andererseits eine transaktionskostenökonomische Argumentation. ${ }^{43}$ Die Transaktionskostentheorie betrachtet die relative Vorteilhaftigkeit institutioneller Arrangements hinsichtlich deren Transaktionskosten. Mit zunehmender Spezifität steigen die Transaktionskosten sowohl für eine marktliche, hybride als auch hierarchische (Eigenerstellung) Koordination, jedoch mit komparativen Vorteilen in Abhängigkeit des Spezifitätsgrades. ${ }^{44}$ Die Spezifität kann sich bspw. auf Humankapitalspezifität bei verwaltungsspezifischen Qualifikationen von Beamten, oder auch auf die Prozessspezifität bestimmter Verfahren des Verwaltungsbezugs beziehen. ${ }^{45}$ Aufgabenfelder mit hoher Spezifität und hoher strategischen Relevanz sollten durch den Staat und seine öffentlichen Verwaltungen wahrgenommen werden. Für alle anderen ist die ökonoomische Vorteilhaftigkeit einer Fremdvergabe zu prüfen. ${ }^{46}$ Von einer hohen strategischen Relevanz wird bspw. bei Aufgaben von Verfassungsrang und Aufgaben mit zentraler Bedeutung für das Gemeinwohl ausgegangen. ${ }^{47}$

PPPs bewegen sich im Bereich zwischen öffentlicher Ausschreibung (als SpotTransaktion) und vertikaler Integration (Eigenerstellung). ${ }^{48}$ Die Anwendbarkeit von PPPs ist in der Rechtssprechung klar geregelt und Grenzen gesetzt. ${ }^{49}$ Für die Bereitstellung öffentlicher Güter obliegt der öffentlichen Hand eine besondere Verantwortung. ${ }^{50}$ Mangels Durchgriffsmöglichkeit und Steuerungseinfluss gegenüber dem privaten Partner scheiden rein marktliche Lösungen aus. ${ }^{51}$ Die Auswahl des privaten Partners erfolgt in der Regel auf Basis des Vergaberechts, d.h. mittels Competitive Tender-Verfahren, oder bei „besonders komplexen Aufträgen“ im Wettbewerblichen Dialog. ${ }^{52}$ Konzepten der Privatwirtschaft folgend sieht sich der Staat bzw. die Verwaltung bei besonders komple-

40 Vgl. Borins/Grüning (1998), S. 30, „marktähnliche Mechanismen (MÄM)“; Harms/Reichard (2003), S. 15, „Hybridisierung“; Roggenkamp (1999), S. 34, „intermediärer Bereich“.

41 Vgl. Macneil (1978), S. 854 ff., neoklassische und relationale Verträge.

42 Vgl. Eichhorn (1995), S. 175; Budäus (2003), S. 220 f., Budäus unterscheidet zwischen „PPP in einem engeren Sinn“ und „PPP in einem weiteren Sinn“; Kommission der europäischen Gemeinschaften (2004), S. 9, PPP auf „Vertragsbasis“" und ,institutionalisierte“ PPP.

43 Vgl. zur strategischen Bedeutung Picot (1991), S. 346; vgl. zu Transaktionskosten Coase (1937); Williamson (1991a), S. $281 \mathrm{ff}$.

44 Vgl. Williamson (1991a), S. 284.

45 Vgl. Lenk (2004), S. 24.

46 Vgl. Budäus (1998), S. 4.

47 Vgl. Lenk (2004), S. 24.

48 Vgl. Batran/Eßig/Schäfer (2004), S. 134.

49 Vgl. § 65 (1), Nr. 1 BHO „Der Bund soll sich ... an der Gründung eines Unternehmens nur beteiligen, wenn ein wichtiges Interesse des Bundes vorliegt und sich der vom Bund angestrebte Zweck nicht besser und wirtschaftlicher auf andere Weise erreichen lässt ....”.

50 Vgl. zur Problematik öffentlicher Güter Savas (2000), S. 44 ff.; Borins/Grüning (1998), S. 28 f.

51 Vgl. Budäus/Grüning (1997), S. 28 und $§ 65$ (1), Nr. 3 BHO „... der Bund einen angemessenen Einfluss, insbesondere im Aufsichtsrat oder in einem entsprechenden Überwachungsorgan erhält, ...".

52 Vgl. Abbildung 1, Artikel 29 (1), Richtlinie 2004/18/EG. 
xen Lösungen lediglich in der Rolle desjenigen, der gewünschte Outputs definiert. Das konkrete Konzept, die Umsetzung, Betrieb und evtl. Wartung liegen beim privaten Partner. ${ }^{53}$ Neben möglichen Effizienzgewinnen durch die Nutzung von Marktmechanismen bei der öffentlichen Aufgabenerstellung liegen kurzfristige Vorteile von PPPs in der Verschiebung von (Investitions-) Ausgaben auf den privaten Bereich. Langfristig stellt dies nur eine zeitliche Verlagerung von Zahlungsströmen dar, indem für von Privaten finanzierte und betriebene Einrichtungen Gebühren vom Staat bezahlt werden müssen. ${ }^{54}$ Dies mag ein Grund sein, weshalb anfallende Gebühren häufig direkt beim Benutzer erhoben werden (bspw. Maut bei privatisierten Autobahnen). Der ökonomische Erfolg von PPPs hängt entscheidend von einer sorgfältigen ökonomischen Analyse der Beschaffungssituation und nicht zuletzt aufgrund der entstehenden Abhängigkeit von einer sorgfältigen Auswahl des privaten Partners ab. Aus dem Private SCM sind eine Vielzahl operativ und strategisch ausgerichteter Lieferantenbewertungskonzepte bekannt. ${ }^{55}$ Die EU-Kommission stellt mit ihrem Grünbuch erste Überlegungen auf der Basis einer neuen EURichtlinie zu - dort so genannten - Öffentlich-Privaten Partnerschaften zur Diskussion. ${ }^{56}$

\section{Zur Notwendigkeit eines Public Supply Chain Management aus Sicht des Supply Chain Management}

Die Idee, nicht Einzelinstitutionen, sondern gesamte Wertschöpfungsketten zu steuern, steht im Mittelpunkt des Supply Chain Management (SCM). Dabei handelt es sich im Kern um einen betriebswirtschaftlichen Ansatz. Analyseobjekt der Betriebswirtschaftslehre ist klassischerweise das „Wirtschaften in Betrieben“. Wirtschaften steht dabei für den Umgang mit Knappheit, Betriebe für Institutionen zur Deckung von Fremdbedarfen. Tatsächlich deckt den Bedarf des Endkonsumenten i.d.R. nicht mehr ein Einzelunternehmen (,Betrieb“), sondern eine Kette mehr oder weniger miteinander verwobener Unternehmen („Kette“bzw. „Chain“). Hauptgrund dafür sind die Effizienzvorteile von Spezialisierung, was zur Reduzierung der Fertigungstiefe eines Einzelbetriebs führt. Dies lässt sich empirisch leicht über den Fremdbezugsanteil nachweisen, der bei Industriebetrieben i.d.R. heute bereits bei weit über $50 \%$ liegt. $^{57}$

Die Supply Chain ist also nichts anderes als ein Unternehmensnetzwerk, das von der Rohstoffgewinnung bis zur Endproduktherstellung bzw. Endproduktdistribution reicht; Supply Chain Management steht für ein Führungskonzept zur Gestaltung dieses Netzwerks. An die Stelle des Erfahrungsgegentandes „Einzelunternehmen“ tritt der Erfahrungsgegenstand ,unternehmensübergreifende Zusammenarbeit“; an die Stelle eines Er-

53 Vgl. Parker/Hartley (2003), S. 98. Beispielsweise werden für die IT-Benutzerberatung messbare Kriterien, wie Reaktionszeit usw. vorgegeben, ohne dabei dem potenziellen privaten Anbieter organisatorische und konzeptionelle Vorgaben zu machen.

54 Parker/Hartley (2003), S. 98, ,future leasing costs“, vgl. Budäus (2003), S. 225, Finanzierungs- und Betreibermodelle für Infrastrukturprojekte sowie Eichhorn (1995), S. 175 f.; Greiling (1994), S. 74.

55 Vgl. Wagner (2001), S. 201 ff.; Eßig (2003), S. 323 ff., „Supplier Lifetime Value“.

56 Vgl. Kommission der europäischen Gemeinschaften (2004).

57 Vgl. Dobler/Burt/Starling (2003), S. 40; Eßig (2005), S. 4. 
kenntnisgegenstandes „Wirtschaften in Betrieben“58 tritt der Erkenntnisgegenstand „Wirtschaften in Netzwerken“.

\section{Zur Notwendigkeit eines Public Supply Chain Management aus Sicht von New Public Management}

Tatsächlich ist das Denken in Netzwerken für den öffentlichen Sektor weitaus typischer als für private Einzelunternehmen. Das Zusammenspiel unterschiedlicher Institutionen aus Politik, Administration und öffentlicher Unternehmen erfordert differenzierte Managementstrukturen. ${ }^{59}$ Aktuelle Weiterentwicklungen werden unter dem Oberbegriff New Public Management (NPM) diskutiert. Eine Einteilung des Sammelbegriffs NPM lässt sich auf drei wesentliche Analyseebenen konzentrieren. Die erste Ebene (makroökonomische Reformen) befasst sich mit dem grundlegenden Funktionswandel des Staates. Die Frage nach den originären Kompetenzen ${ }^{60}$ des Staates führt zu einem Zurückdrängen der Staatstätigkeit. ${ }^{61}$ Im Konzept des Gewährleistungsstaates trägt der Staat bzw. die öffentliche Verwaltung die Verantwortung für die Sicherstellung der Leistungserbringung, ohne dabei selbst die eigentliche Ausführung zu übernehmen. ${ }^{62}$ Dabei spielt das institutionelle Arrangement der Zusammenarbeit eine besondere Rolle. Aufgrund der Gewährleistungspflicht scheiden rein marktliche Lösungen häufig ex ante bereits aus, so dass hybride Formen der Zusammenarbeit (bspw. in Form von PPPs) mit ausgeprägten Regelungsund Kontrollmechanismen eine wesentliche Rolle spielen. ${ }^{63}$ Der Steuerung zwischen öffentlicher Eigenerstellung und Marktbezug werden „marktähnliche Mechanismen“ zuerkannt. ${ }^{64}$ Die Frage nach der optimalen Leistungstiefe ${ }^{65}$ des Staates ist verbunden mit einer Zusammenarbeit mit dem privaten Sektor und beeinflusst damit auch die mikroökonomische Reformebene. ${ }^{66}$ Darunter fallen zum einen Aspekte der Public Governance, die die Größe, Abgrenzung und Relevanz des öffentlichen Sektors als Ganzes thematisieren. ${ }^{67}$ Zum anderen gehört dazu die Binnen- bzw. Mikro-Perspektive des Public Management, welche in erster Linie Reformanstrengungen in der Verwaltungspraxis unterstützt. ${ }^{68}$ Organisationsexternes Makromanagement und organisationsinternes Mikromanagement weisen in zweierlei Hinsicht Parallelen zum Private SCM auf. Das Mikromanagement befasst sich mit Fragen stärkerer „Kunden-“ bzw. Bürgerorientierung, also der bewussten Ausrichtung der Leistungsangebote auf den Nachfrager (z.B. Öffnungszeiten

58 Vgl. Schweitzer (2004), S. 24.

59 Vgl. Erridge/McIlroy (2003), S. 549, Netzwerk als Beziehungsgeflecht.

60 Vgl. Prahalad/Hamel (1990), Kernkompetenzen.

61 Vgl. Hammerschmid (2001), S. 52, Eichhorn (2001), S. 411, „Lean Government“.

62 Vgl. zum Konzept des Gewährleistungsstaates Schedler/Proeller (2003), S. 35 ff.; Reichard (2004), S. 48 ff.

63 Vgl. Ebers/Gotsch (2002), S. 233 f.; Williamson (1991a), S. 281 sowie Göbel (2004), S. 19. Die Möglichkeit der administrativen Kontrolle konstituiert sich bspw. auch am Streikverbot für Beamte (Hierarchie).

64 Vgl. Borins/Grüning (1998), S. 30.

65 Vgl. Naschold u.a. (2000), S. $17 \mathrm{ff}$.

66 Zweite Analyseebene, vgl. Budäus (1998), S. 4; Hammerschmid (2001), S. 52 f.; Batran/Eßig/Schäfer (2004), S. 128.

67 Zur Makro-Perspektive vgl. Hammerschmid (2001), S. $51 \mathrm{ff}$.

68 Vgl. Hammerschmid (2001), S. 51 ff.; Budäus (1998), S. 3 ff. 
von Behörden, Schnelligkeit der Antragsbearbeitung usw.). Des Weiteren betrifft das Makromanagement das Außenverhältnis des öffentlichen Sektors und dessen (politische) Steuerung. Hierbei stehen Fragenstellungen im Mittelpunkt, wie eine Einbindung des privaten Sektors erfolgen kann und wie Lieferbeziehungen (private-public) optimal zu gestalten sind. ${ }^{69}$ Mit dieser Zweiteilung wird eine weitere Parallele zwischen SCM und NPM deutlich: SCM hat ebenfalls eine Binnen- und eine Makro-Perspektive. Einerseits ist es ein betriebswirtschaftliches Konzept, das zur Optimierung der einzelnen Elemente einer Wertschöpfungskette, d.h. den beteiligten Unternehmen, beitragen soll. Andererseits ist SCM an der Gesamtkette, eben am Wirtschaften in Netzwerken, orientiert. ${ }^{70}$

\section{Das Grundmodell: Wider einer unreflektierten Übernahme des privatwirtschaftlichen Supply Chain Management-Ansatzes}

Nicht zu unrecht wird davor gewarnt, betriebswirtschaftliche Ansätze zur Führung privater Betriebe unreflektiert auf öffentliche Institutionen zu übertragen. ${ }^{71}$ Andererseits zeigt die öffentliche Betriebswirtschaftslehre, dass sehr wohl ökonomische Konzepte sinnvoll einsetzbar sind. Wenn wir deshalb in der Folge den Ansatz des Public Supply Chain Management entwickeln, versuchen wir, den Besonderheiten des Analyseobjekts „Public Supply Chain“ im Verhältnis zur privatwirtschaftlichen Supply Chain gerecht zu werden. Dazu werden wir in der Folge (a) die Gemeinsamkeiten bzw. Unterschiede des Analyseobjekts herausarbeiten und (b) im nächsten Schritt die Übertragung der Konzeptmerkmale des Supply Chain Management auf das Public Supply Chain Management vornehmen.

1. Analyseobjekte Public Supply Chain und unternehmerische Supply Chain im Vergleich

Obwohl - oder besser: weil - Supply Chain Management in den letzten Jahren zu den „boomenden“ Teildisziplinen der Betriebswirtschaftslehre gehört, ist häufig von einer reinen „Modeerscheinung ${ }^{\text {“72 }}$ die Rede. Um diesem Vorwurf zu begegnen, ist es erforderlich, das Analyseobjekt vor der Entwicklung der Konzeptmerkmale genau zu definieren. Wir haben bereits in Abschnitt 1.2 die mit SCM verbundene Verschiebung der Analyseebene vom Einzelunternehmen zum Netzwerk skizziert. Netzwerk steht in diesem Fall für mehr als zwei privatwirtschaftliche Unternehmen. Public Supply Chain Management stellt daher ebenfalls das Netzwerk in den Mittelpunkt der Analyse.

69 Vgl. Hammerschmid (2001), S. 53 und S. 55; Budäus (1998), S. 4.

70 Vgl. Mentzer u.a. (2001), S. 2 und 6 f. Neher (2003), S. 30 ff. versteht unter dem Supply Chain Value die Erweiterung der intraorganisatorischen Betrachtungsperspektive durch eine Supply Chain Perpsektive. Im Kern geht es um eine langfristige Maximierung des individuellen Unternehmenswertes unter Berücksichtigung der Zielsetzungen auf der Supply Chain Ebene.

71 Vgl. Borins/Grüning (1998), S. 40; Eichhorn (2001), S. 410.

72 Vgl. Göpfert (2002), S. 27; Müller/Seuring/Goldbach (2003), S. 419. 
Tatsächlich ist dieses Netzwerk im Rahmen des Public SCM institutionell weitaus umfangreicher und komplexer als im privatwirtschaftlichen SCM. Eichhorn (2001) hat eindrucksvoll deutlich gemacht, dass die öffentliche Betriebswirtschaftslehre im Gegensatz zur „kommerziellen“ Betriebswirtschaftslehre eine Vielzahl von Objekten untersucht, die zwar auch kommerzielle Unternehmen erfassen kann (sofern sie mit öffentlichen Aufgaben beliehen werden), darüber hinaus aber auch bzw. vor allem Gebietskörperschaften, Non-Profit-Betriebe, öffentliche Vereinigungen und andere mehr. ${ }^{73}$ Wertschöpfungsketten im öffentlichen Sektor stellen ein System aus ,gesellschaftlichen, politischen, ökonomischen und technischen Aktivitäten ${ }^{\text {(77 }}$ dar. Das bedeutet, dass das Netzwerk als Analyseobjekt des Public SCM faktisch ein Mehr-Ebenen-Netzwerk ist, wie es in Abbildung 3 dargestellt ist: Es umfasst (1) die Ebene privatwirtschaftlicher Unternehmen, die entweder im Besitz des öffentlichen Sektors sind oder von ihr Aufträge erhalten, (2) die Ebene der öffentlichen Verwaltungen (exekutive Administration wie Behörden, Sozialversicherungen, Verbände) sowie (3) die Ebene der politischen Entscheidungsinstitutionen (gesetzgebende bzw. rahmenbestimmende Vorgaben). Beim Public SCM tritt zur Analyse von Intra-Netzwerk-Beziehungen noch die Analyse von Inter-Netzwerk-Beziehungen. So geht es bspw. nicht nur um die Frage, welche Unternehmen bei der Leistungserstellung zusammenarbeiten, sondern wie diese mit Entscheidungsträgern und Leistungserbringern auf anderen Ebenen verknüpft sind. Beispielhaft sei die Müllentsorgung genannt, die möglicherweise von einem kommerziellen Unternehmen durchgeführt wird, das die Kommunalverwaltung im Rahmen einer Vergabe ausgewählt hat bzw. laufend überwacht und dessen „Entlohnung“ in Form der Müllgebühren durch das Kommunalparlament (als demokratischer Vertreter des „Endkunden“ Bürger) festgelegt wird.

Tatsächlich bedeutet das, dass die im Rahmen des Public SCM untersuchten Einzelinstitutionen (Netzwerkmitglieder) nicht originär ökonomische Institutionen (im Sinne der Deckung von Eigen- oder Fremdbedarfe wie Haushalte und Unternehmen) sind. Trotzdem ist es möglich, eine betriebswirtschaftliche Analyseperspektive einzunehmen. ${ }^{75}$ Ohne an dieser Stelle die Diskussion aufzugreifen, ob die Betriebswirtschaftslehre tatsächlich dem Primat der Ökonomie zuzuordnen ist - oder stärker durch psychologische (bspw. Markt- und Werbepsychologie), soziologische (bspw. Gruppenverhalten im Betrieb) oder Erkenntnisse anderer Disziplinen beeinflusst wird, plädieren wir im Kern für eine ökonomisch fundierte Institutionenlehre des Public SCM. ${ }^{76}$ Dies bedeutet zweierlei:

- Erstens werden die im Netzwerk beteiligten Institutionen aus ökonomischer Perspektive untersucht. Sie tritt ausdrücklich neben rechtswissenschaftliche, politikwissenschaftliche oder andere Analyseperspektiven. ${ }^{77}$ Damit handelt es sich um das Wissenschaftsprogramm der modernen Institutionenökonomik, das auch nichtwirtschaftliche Gegenstandsbereiche wie Recht ${ }^{78}$ oder Politik (Neue Politische

73 Vgl. Eichhorn (2001) S. 409.

74 Thom/Ritz (2003), S. 441.

75 Vgl. ähnlich Göbel (2004), S. 3-5.

76 Vgl. zum Primat der Ökonomie bspw. Bayón (1997) sowie Schneider (1983).

77 Vgl. Göbel (2004), S. 4.

78 Vgl zur ökonomische Analyse des Rechts Adams (2004). 
Ökonomik) einer ökonomischen Analyse unterzieht. ${ }^{79}$ Es werden also nicht Verhaltensmodelle der Psychologie, Soziologie oder anderer Disziplinen, sondern der homo oeconomicus als typisches Verhaltensmodell der Ökonomik zugrunde gelegt.

- Zweitens ist das (Mehr-Ebenen-) Netzwerk selbst eine ökonomische Institution; ${ }^{80}$ im Verständnis der Neuen Institutionenökonomik eine hybrid, d.h. gemischt marktlich-hierarchisch gesteuerte Institution. ${ }^{81}$ Die am Netzwerk beteiligten Parteien sind im Prinzip voneinander unabhängig (Außenbeziehung Markt), arbeiten aber im Rahmen der Leistungsgestaltung und -erstellung einer gemeinsamen Endleistung zusammen. Die Frage, ob und wenn ja welche Führungsprozesse und -maßnahmen für dieses Gesamtnetzwerk erforderlich sind, steht im Mittelpunkt des Public SCM. Referenzpunkt für diese Managementprozesse ist das Private SCM, welches in der Zwischenzeit schon einen gewissen Reifegrad erreicht hat. Das Mehr-Ebenen-Netzwerk erfordert jedoch die Weiterentwicklung der Konzeptmerkmale des Public SCM.

79 Vgl. Göbel (2002), S. 32; Lenk (2004), S. 22 ff. und Richter (1994).

80 Vgl. Picot/Dietl/Franck (2002), S. 11 und S. 206 ff.

81 Vgl. Williamson (1990), S. 16 ff. sowie Williamson (1991b), S. 23-25. 


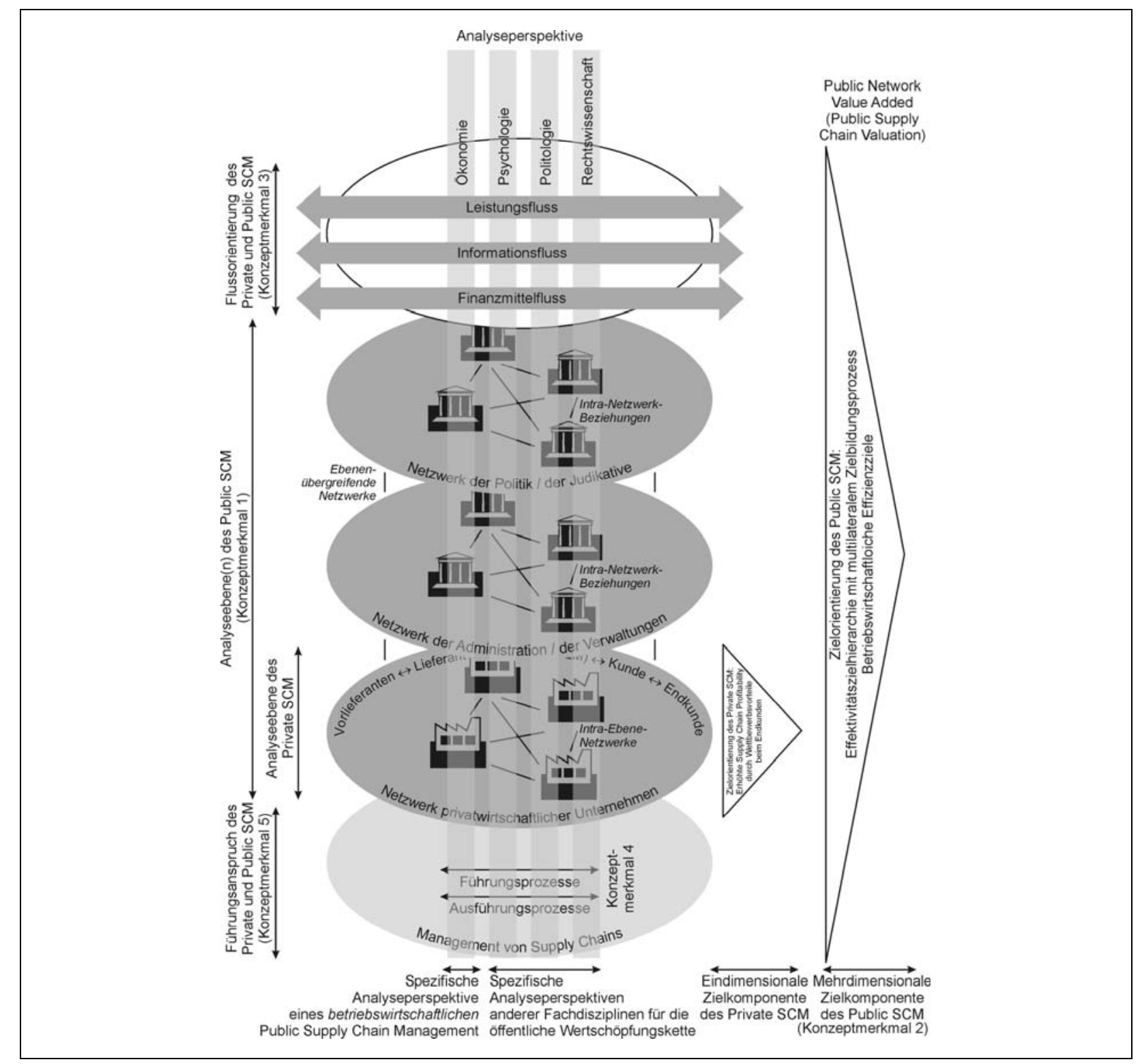

Abb. 3: Analyseebenen und Konzeptmerkmale des Public Supply Chain Management Quelle: Eigene Darstellung

2. Entwicklung von Konzeptmerkmalen eines Public Supply Chain Management

Konzeptmerkmal (1): Netzwerkorientierung

Wir haben bereits bei der Darstellung des Analyseobjekts auf die grundlegende Orientierung an Netzwerkstrukturen hingewiesen. Das Erkenntnisinteresse umfasst also nicht lediglich bilaterale Beziehungen zwischen zwei beteiligten Parteien, sondern mehr als zwei Institutionen, die vertikal und/oder horizontal miteinander verflochten sind, um gemeinsam Wertschöpfung zu erbringen.

„Supply Chain“ steht mithin für dieses Netzwerk, was vor dem Hintergrund der Wertschöpfungsorientierung semantisch nicht unproblematisch ist. Begriffe wie (Wert-) Kette, Netzwerk, Supply Chain und Supply Network werden mehr oder weniger undifferenziert 
gleichgesetzt. ${ }^{82}$ „Supply“ ist als Terminus insbesondere in der Beschaffungsliteratur zu finden, als er suggeriert und insofern missverständlich, es würde nur der versorgungsseitige Teil einer Gesamtkette untersucht. ${ }^{83}$ Wie wir nachfolgend im Rahmen des Konzeptmerkmals Zielorientierung noch zeigen, ist die explizit durch den Endverbraucher der Gesamtleistung gesteuerte Kette das Ziel eines „echten“ (Private und Public) Supply Chain Management. Der semantisch korrekte Vorschlag, aus diesem Grund „Supply“ durch „Demand ${ }^{\text {‘64 }} \mathrm{zu}$ ersetzen, hat sich allerdings nicht durchgesetzt. ${ }^{85}$

Ähnliches gilt für die Idee, den eigentlich durch vertikale Ausrichtung besetzten Begriff „Chain“ bzw. „Kette“ durch „Net“ bzw. „Netzwerk“ abzulösen. ${ }^{86}$ Faktisch sind die Institutionen der Public Supply Chain sowohl vertikal als auch horizontal verwoben; so arbeiten Behören einer Gebietsebene gleichrangig zusammen und andererseits sind sie vertikal politischen Entscheidungen nachgelagert bzw. einem möglichen Erfüllungsgehilfen der Privatwirtschaft vorgelagert.

Noch schwieriger wird es, wenn wir den gesamten Term „Public Supply Chain“ faktisch als „öffentliches Wertschöpfungsnetzwerk“ verstehen. Das bedeutet zum einen eine eindeutige Abgrenzung öffentlicher von privaten Aufgaben und zum anderen die Übertragung des volks- bzw. betriebswirtschaftlichen Wertschöpfungsbegriffs auf den öffentlichen Sektor:

a) Vereinfacht sind öffentliche Aufgaben aus öffentlichem Interesse abgeleitet (vgl. Abbildung 4). Konkret bedeutet das die Erstellung von Leistungen, die einem gesellschaftlich gewünschten Ziel dienen. Beispielhaft sei eine dem Gemeinwohl dienende hohe Lebenserwartung der Bevölkerung genannt. ${ }^{87}$ Daraus sind Zielsetzungen der Bildungs-, Ernährungs- und Gesundheitspolitik abzuleiten, in entsprechenden Gesetzen bzw. Verordnungen als öffentliche Aufgaben zu formulieren und schließlich in Schulen, bei Gesundheitsämtern, in Sportzentren, durch Krankenhäuser mit privater oder öffentlicher Trägerschaft, in der pharmazeutischen Industrie sowie vielen anderen Institutionen administrativer wie privatwirtschaftlicher Art umzusetzen. Abbildung 4 verdeutlicht in diesem Zusammenhang einmal mehr das Mehr-Ebenen-Netzwerk, das beim Public SCM im Gegensatz zum Private SCM zum Einsatz kommt. ${ }^{88}$ Die politische Steuerungsebene (Netzwerkebene der Politik) in Form von Exekutivspitze und Legislative gibt nachgeordneten Verwaltungseinheiten den Orientierungsrahmen vor. ${ }^{89}$ Ergebnis dieser Politikformulierung sind politische Programme, d.h. Gesetze, Haushaltsentscheidungen usw., die im Rahmen der Politikdurchführung von öffentlichen und privaten Aufgabenerfüllern zur Erfül-

82 Vgl. Marbacher (2001), S. 16 ff., dagegen Corsten/Gössinger (2001), S. 82 ff.

83 Vgl. Arnold u.a. (2005), S. 43 f.

84 Vgl. Corsten (2002), S. 947; Corsten/Gössinger (2001), S. 85 und Marbacher (2001), S. 20 f.

85 Vgl. Busch/Dangelmaier (2002), S. 4; Halldorsson/Skjott-Larsen/Kotzab (2003), S. 33 und Zimmermann (2003), S. 13.

86 Vgl. Busch/Dangelmaier (2002), S. 4.

87 Vgl. Eichhorn (2001), S. 410.

88 Vgl. auch Grünenfelder (1997), S. 61 f. „Verantwortungs-Ketten-Diagramm“, Naschold u.a. (2000), S. 39 ff., „Leistungskette“.

89 Vgl. Thom/Ritz (2003), S. 438. 
lung öffentlicher Aufgaben angewendet werden (müssen). ${ }^{90}$ Die administrative Netzwerkebene ist für die konkrete Umsetzung der auf der politischen Steuerungsebene festgelegten Zielsetzungen verantwortlich. ${ }^{91}$ Die Unterscheidung in politische Ziele, öffentliche Aufgaben und öffentliche und/oder private Leistung (Aufgabenerfüllung) spielt eine entscheidende Rolle bei der Frage nach der Gewährleistungsverantwortung. ${ }^{92}$ Interdependenzen zwischen den Steuerungsebenen werden anhand von Output (Leistung der betrieblichen Steuerungsebene), Impact (Wirkung politischer Zielsetzung als Reaktion der „Betroffenen“) und Outcome (gesellschaftliche Auswirkungen der jeweiligen Politik) differenziert. ${ }^{93}$ Dabei ist die administrative Ebene nicht für den Outcome verantwortlich, da dieser externen Einflüssen unterliegt und grundlegend von der politischen Steuerungsebene geprägt wird. ${ }^{94}$

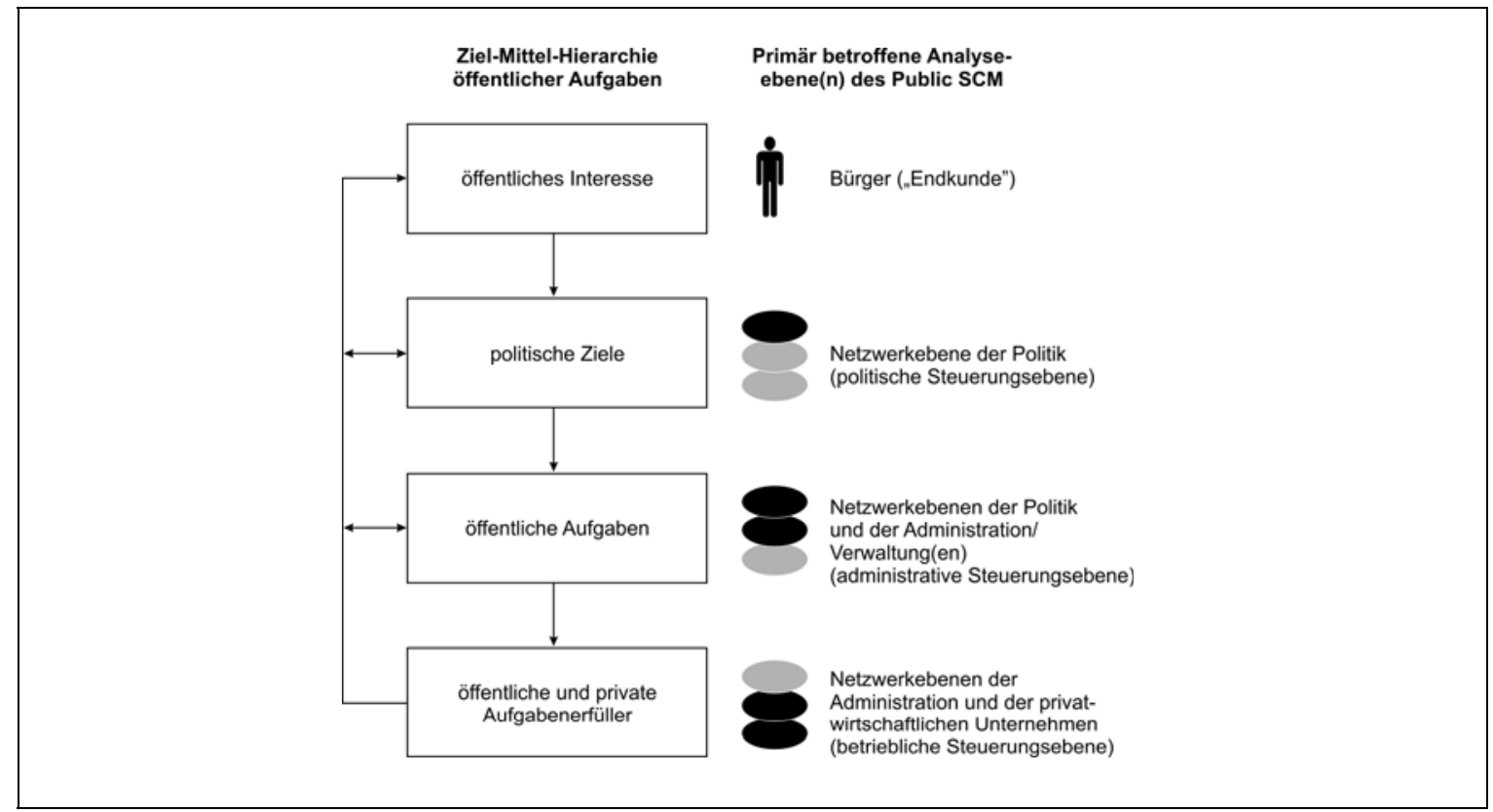

Abb. 4: Öffentliche Aufgaben

Quelle: $\quad$ Eigene Darstellung in Anlehnung an Eichhorn (2001), S. 410; Thom/Ritz (2003), S. 438

b) Wertschöpfung ist demzufolge bei öffentlichen Aufgaben erbracht, wenn sie der Erfüllung von in öffentlichem Interesse liegenden Zielen dienen. Der Wertschöpfungsbegriff des Private SCM lehnt sich an eine volks- und betriebswirtschaftliche Wertschöpfungsdefinition an. Demzufolge ist Wertschöpfung im statischen Sinne die Differenz aus der Bruttowertschöpfung eines Wirtschaftsbereiches abzüglich der Vorleistungen aus anderen Wirtschaftsbereichen bzw. auf einzelbetrieblicher Ebene Erlöse abzüglich Vorleistungskosten. ${ }^{95}$ Wertschöpfung kann damit aus ökonomischer Sicht auf zwei Arten gesteigert werden, durch Erhöhung der Erlöse und/oder

90 Vgl. Naschold u.a. (2000), S. 50.

91 Vgl. Thom/Ritz (2003), S. 439; Budäus (2003), S. 215, Gewährleistungsverantwortung.

92 Vgl. Naschold u.a. (2000), S. 56.

93 Vgl. Grünenfelder (1997), S. 58 und S. 61 f.; Naschold u.a. (2000), S. 50 f. und Thom/Ritz (2003), S. 439.

94 Vgl. Thom/Ritz (2003), S. 439.

95 Vgl. Schusser (1999), S. 9 ff. 
durch Senkung der Vorleistungskosten. Der dynamische Wertschöpfungsbegriff setzt am Prozess der Leistungsentwicklung und -erfüllung an. Im öffentlichen Sektor muss dieser Prozess einen gesellschaftlichen und politikrelevanten Zusatznutzen schaffen. Nur dann ist die politische Führung bereit, Mittel bereitzustellen. ${ }^{96}$ Allgemein wird Wertschöpfung erbracht, wenn die Zahlungsbereitschaft des Endkonsumenten die Kosten der Leistungserstellung übersteigt. Die Übertragung dieses Prinzips auf öffentliche Wertschöpfungsketten ist nicht trivial, da i.d.R. das Besteuerungsprinzip herrscht, d.h. keine schlüssigen Tauschbeziehungen und damit keine direkten Verbindungen zwischen Leistung und Gegenleistung (Bezahlung) bestehen. ${ }^{97}$ Man denke nur an Sozialversicherungssysteme mit Solidarprinzip (Krankenversicherung) oder an steuerfinanzierte Staatsaufgaben wie Hochschulbildung bei denen die direkte Leistungsabgabe unentgeltlich erfolgt. ${ }^{98}$ Larisch (1999) entwickelt für den Austausch sozialer Dienstleistungen ein System aus Empfänger (z.B. Patient), Erbringer einer Leistung (Ambulanter Pflegedienst) sowie Bedarfsfeststeller (Arzt), Kostenträger (Krankenversicherung/-kasse) und Anspruchgestalter (staatliche Festlegung von gesetzlichen Mindeststandards) zur Koordination des inhärent komplexen Beziehungsgeflechts. ${ }^{99}$ Soziale Dienstleistungen typologisiert Hamman (2004) als „Dienste von Menschen an Menschen“ (Output als soziale Dienstleistung, daneben „Dienste von Menschen für Menschen“, Output vorwiegend als Sachleistung) und kritisiert dabei die mangelnde Dienstbereitschaft, d.h. die freiwillige Dienstleistungserstellung der Bürger. Ökonomisch betrachtet liegt hier ebenfalls Marktversagen vor, das die Rolle des Gewährleistungsträgers wiederholt deutlich herausstellt. ${ }^{100}$

Festzuhalten bleibt, dass das konstituierendes Konzeptmerkmal des Public SCM die Netzwerkorientierung ist. Im Gegensatz zum Private SCM handelt es sich um ein MultiEbenen-Netzwerk. Der Begriff „Public Supply Chain“ steht für „öffentliches Wertschöpfungsnetzwerk“, d.h. es dient der Erfüllung öffentlicher Aufgaben bei höchstmöglicher Wertschöpfung. Wie sich diese Wertschöpfung operationalisieren lässt, soll im Rahmen des Konzeptmerkmals Zielorientierung diskutiert werden.

Konzeptmerkmal (2): Zielorientierung

Thom und Ritz (2003) schlagen bei der Betrachtung des Leistungsprozesses vor, die erbrachten Outputs nicht primär auf die Nachfrager und deren Zahlungsbereitschaft auszurichten. ${ }^{101}$ Vielmehr ist die Einflussnahme auf die Adressaten der Leistung (Impact) zur „Erzielung einer gesamtgesellschaftlichen Wirkung“ (Outcome) von Bedeutung. Das Ziel ist eine langfristige Verbesserung der gesellschaftlichen Rahmenbedingungen. ${ }^{102}$ Dieser Argumentation folgt die Kritik an der Schätzung von Zahlungsbereitschaften. ${ }^{103}$ Werden

96 Vgl. Thom/Ritz (2003), S. 441.

97 Vgl. Eichhorn (1987) S. 218.

98 Fehlender Preismechanismus, vgl. Eichhorn (1987), S. 219 sowie Thom/Ritz (2003), S. 441.

99 Vgl. Larisch (1999), S. 137.

100 Vgl. Hammann (2004), S. 90 f.

$101 \mathrm{Vgl}$. Thom/Ritz (2003), S. 441.

$102 \mathrm{Vgl}$. Thom/Ritz (2003), S. 441, Gesellschaftsbedarf und Nutzensteigerung.

103 Vgl. Brümmerhoff (2001), S. 202. 
Leistungen nicht marktmäßig bereitgestellt, existieren keine Preisvorstellungen. So sind bei öffentlichen Gütern Individuen nicht in der Lage, Preise zu bestimmen bzw. Maßnahmen in monetäre Größen zu übertragen (bspw. Landesverteidigung). Gerade wegen der scheinbar schwierigen Bewertung öffentlicher Leistungen aus wohlfahrtsökonomischen Überlegungen heraus mag diese Kritik zutreffend sein, jedoch werden durch diese Abstrahierung eine betriebswirtschaftliche Analyse und eine Ableitung von Gestaltungsempfehlungen deutlich erschwert.

Das Gesamtziel höchstmöglicher Wertschöpfung durch aktive Gestaltung der Supply Chain ist für privatwirtschaftliche Supply Chains insofern leichter zu operationalisieren, als dass alle Beteiligten kommerzielle Unternehmen sind und damit individuell dasselbe Ziel der Gewinnmaximierung verfolgen. Chopra und Meindl (2001) sehen deshalb als Oberziel des Private SCM die Erhöhung der „Supply Chain Profitability“. ${ }^{104}$ Weiterhin haben Einzelwirtschaften als Oberziel die Maximierung finanzwirtschaftlicher Kennzahlen (bspw. Economic Value Added, Shareholder Value, Return on Investment), welche auch für privatwirtschaftliche Netzwerke als Erkenntnisobjekte des Private SCM Anwendung finden. Möller (2003) spricht vom Ziel des Supply Network Value Added und dem dazu erforderlichen Prozess der Supply Chain Valuation. Damit ist explizit der Finanzmittelfluss der Supply Chain angesprochen. ${ }^{105}$

Für das Public SCM greifen wir auf den oben definierten Wertschöpfungsbegriff einerseits und auf die auch beim Private SCM übliche Unterscheidung zwischen Supply Chain-Effizienz- und Supply Chain-Effektivitätsziel zurück. ${ }^{106}$ Das Effektivitätsziel setzt an der Ebene politischer Ziele an, die in demokratischen Verfahren festgelegt werden und in der Regel kurzfristig nicht veränderbar sind. ${ }^{107}$ Regierung und Parlament geben somit Ziele vor („Was“), welche durch die Verwaltungen möglichst effizient umgesetzt werden sollen (,Wie“). ${ }^{108}$ Das Effizienzziel (,Maßnahmenebene“(109) stellt die Rationalisierung von Supply Chain-Prozessen in den Vordergrund und setzt damit an der Kostenseite an. Im Private SCM wird dieses Ziel - entsprechend der logistisch geprägten Tradition des SCM - i.d.R. aus internen Vorgaben bspw. zu Kosten- oder Durchlaufzeitenreduzierungen oder zum Bestandsabbau operationalisiert, ${ }^{110}$ meist in Form eines Lösungsbeitrags zum Forrester- bzw. Bullwhip-Effekt. ${ }^{11}$ Für das Public SCM ist eine hohe Analogie erkennbar - selbstverständlich sollen auch öffentliche Aufgaben möglichst effizient (d.h.

104 Vgl. Chopra/Meindl (2001), S. 6.

105 Vgl. Möller (2003), S. 71.

106 Vgl. Bechtel/Jayaram (1997), S. 18 sowie Buscher (1999), S. 450 f.

107 Vgl. Budäus, (1994), S. 59 sowie Schedler/Proeller (2003), S. 35.

108 Vgl. Schedler/Proeller (2003), S. 54.

109 Budäus (1994), S. 59.

110 Vgl. Kuhn/Hellingrath (2002), S. 10 und S. 15.

111 Vgl. Göpfert (2002), S. 33 f. und Wagner (2001), Weiterhin befassen sich innerhalb des Private SCM Ansätze des Lieferantenmanagements mit Effizienz, indem bspw. durch Bildung von Lieferantenportfolios (-clusterung) eine möglichst transaktionskostenoptimale Zusammenarbeit auf bilateraler Ebene erreicht werden soll. Des Weiteren lässt sich unter Effektivität bspw. die Auswahl geeigneter Lieferanten subsumieren. Morgan/Hunt (1994) untersuchen die Wirkung von Commitment und Vertrauen auf die Beziehungsqualität, woraus sich schließen lässt, dass zur Zielerreichung im Private SCM grundsätzlich nicht nur opportunistische Ergebnismaximierung unterstellt wird. Wie bereits erläutert, sieht das Vergaberecht diese Möglichkeit kaum vor, weshalb die Einschränkung auf den Wertschöpfungsprozess explizit gewählt wurde. 
ein definierter Output mit geringstmöglichem Input bzw. mit definiertem Input ein größtmöglicher Output) erstellt werden. Dieses Prinzip der Wirtschaftlichkeit ist typisch für die (öffentliche) Betriebswirtschaftslehre. ${ }^{12}$ Public SCM kann zu dieser Zielsetzung beitragen, indem es einen Beitrag zur Neustrukturierung und Optimierung des gesamten öffentlichen Wertschöpfungsnetzwerks leistet. Kyrer (2001) sieht gerade in der Bildung eines vertikal und horizontal ausgerichteten Netzwerkes eine zwingende Voraussetzung für die erfolgreiche Umsetzung der Ziele des NPM. ${ }^{113}$ In Anlehnung an Klaus (1999) unterscheiden wir zwischen vertikaler Supply Chain-Integration, horizontaler Supply Chain Integration und hierarchischer Supply Chain-Integration: ${ }^{114}$

- Die sequentielle (vertikale) Public Supply Chain-Integration bestimmt die Tiefe des Netzwerks, d.h. die vertikale Gestaltung der eigentlichen „Kette“. ${ }^{115}$ Dies kann zu einer Reduzierung der Tiefe führen, indem bspw. eine Verwaltungsebene eingespart wird. ${ }^{116}$ Ebenso wäre eine größere Tiefe möglich, wenn die positiven Effekte der Arbeitsteilung bzw. Spezialisierung durch Auslagerung von öffentlichen auf private Aufgabenträger im Rahmen des Outsourcing genutzt werden. Damit stellt sich die Frage des Make-or-Buy öffentlicher Aufgaben neu. ${ }^{117}$ Und schließlich kann die Tiefe im Sinne der Teilnehmerzahl unverändert bleiben und lediglich die Prozessaufgaben neu verteilt werden. Dies ist der Fall, wenn bspw. im Rahmen von Public Private Partnerships die traditionell „öffentliche“ Leistungserstellung (bspw. StraBenbetrieb) in Teilen auf den bereits existierenden privatwirtschaftlichen Partner (bspw. Straßenbauer) vergeben und die Zusammenarbeit zu diesen optimiert wird.

- Die horizontale Public Supply Chain-Integration erfolgt zwischen zwei Institutionen derselben Stufe des Netzwerks bzw. der Kette. Denkbar wäre bspw. die Integration komplementärer Produkte zu einem Leistungsangebot. ${ }^{118}$ Ein typisches Beispiel dafür wären Kommunen und die Bundesagentur für Arbeit, die beide Sozialleistungen erbringen und diese derzeit teilweise im Rahmen des Arbeitslosengeldes II zusammenführen.

- Die hierarchische Public Supply Chain-Integration betrifft die Neuabstimmung zwischen strategischen und taktisch-operativen Planungs- bzw. Ausführungsprozessen. Darunter fiele bspw. die Arbeit der Kommission von Bundestag und Bundesrat zur Modernisierung der bundesstaatlichen Ordnung („Bundesstaatskommission“). Dort sollten die Netzwerkebenen der Politik und der Administration zwischen Bund und Ländern neu strukturiert werden. Allein bei Betrachtung der Netzwerkebene der Politik kommt durch das Scheitern der Kommission die Komplexität und Interessenspluralität des öffentlichen Sektors zum Ausdruck.

112 Vgl. Eichhorn (2001), S. 410.

113 Vgl. Kyrer (2001), S. 54.

114 Vgl. Klaus (1999), S. 123-126.

115 Vgl. Schönsleben/Hieber (2002), S. 52.

116 So sieht das Verwaltungsstruktur-Reformgesetz des Landes Baden-Württemberg eine deutliche Reduzierung der Verwaltungsebenen in der Landesverwaltung vor, in dem bspw. Vermessungsämter in die Landratsämter integriert werden.

117 Vgl. Hahn (2000), S. 15.

118 Vgl. Schönsleben/Hieber (2002), S. 52, Optimierung der „Breite des Netzwerks“. 
Insbesondere bei der hierarchischen Public Supply Chain-Integration ist der Übergang zwischen Effizienz- und Effektivitätsziel bereits fließend. Das Effektivitätsziel konzentriert sich direkt auf die Anforderungen der Endkunden (im Private SCM) bzw. Bürgern (im Public SCM), meist auch als „Supply Chain Management Performance Drivers“ ${ }^{\text {“1 } 19}$ bezeichnet. Im Private SCM wird deshalb die Nachfrage als eigentliches Steuerungsinstrument des SCM angesehen. ${ }^{120}$ SCM ist damit Quelle von Wettbewerbsvorteilen beim Endkunden und verbessert so die Erlösseite des Wertschöpfungsnetzwerks. ${ }^{121}$ Es stellt sich die Frage, wer Endkunde/-konsument des öffentlichen Wertschöpfungsnetzwerkes ist und ob bzw. wie Public SCM zur Kundenorientierung bzw. Erlössteigerung beitragen kann. Im Private SCM kann eine Eingrenzung einfach vorgenommen werden, indem der Kunde derjenige ist, der für die Leistung bezahlt. ${ }^{122}$ Die häufig im öffentlichen Sektor vorzufindenden nicht-schlüssigen Tauschbeziehungen erfordern eine differenziertere Systematik zur Definition des Kunden im Public SCM. Zudem ist der Kunde „Bürger“ häufig durch staatliche Monopole ein Zwangskunde. ${ }^{123}$

Konzeptmerkmal (3): Flussorientierung

Das privatwirtschaftliche SCM-Konzept hat starke Wurzeln in der Logistik. ${ }^{124}$ Es ist daher nicht verwunderlich, dass im Mittelpunkt des Private SCM häufig die Optimierung des unternehmensübergreifenden Leistungs- und zugehörigen Informationsflusses steht. Der Finanzmittelfluss wird teilweise explizit mit einbezogen oder nur in Definitionen genannt, dann aber nicht weiter ausgeführt. ${ }^{125}$

Aus Sicht des Public SCM ist zu beachten, dass viele öffentliche Aufgaben Dienstleistungscharakter haben und der Finanzmittelfluss zwar für die Erlösfrage selbstverständlich hohe Bedeutung hat, aber eine Reihe von Besonderheiten aufweist. Typisch ist das bereits angesprochene Konzept der nicht-schlüssigen Tauschbeziehungen. Larisch (1999) macht am bereits genannten Beispiel sozialer Dienstleistungen deutlich, dass zwischen Leistungserbringer und Leistungsempfänger faktisch nur ein „Quasi-Markt“ herrscht, darüber hinaus aber umfangreiche Leistungs-, Koordinations- und Finanzmittelflüsse mit weiteren Institutionen wie Kostenträger, Anspruchsgestalter und Bedarfsfeststeller existieren. ${ }^{126}$ Der typische Fall des Private SCM, dass der hohe Grad netzwerkinterner Koordination durch den Endkunden (Leistungsempfänger) durch Kauf direkt entlohnt und damit das gesamte Netzwerk unmittelbar belohnt (bzw. im Falle des Nichtkaufs bestraft) wird, ist im Public SCM weitaus komplexer. Geht man davon aus, dass die Gesellschaft, bzw. im Sinne des methodologischen Individualismus die Bürger, „Kunde“ des öffentli-

119 Gattorna/Walters (1996), S. XVI.

$120 \mathrm{Vgl}$. Kotzab (2000), S. 27.

121 Vgl. Arnold/Warzog (2001), S. 20; Eisenbarth (2003), S. 48; Mentzer u.a. (2001), S. 19; Pfohl (1999), S. 194 sowie Tan (2001), S. 41.

$122 \mathrm{Vgl}$. Bandemer (2001), S. 419.

123 Vgl. Bandemer (2001), S. 419, bspw. Einwohnermeldeamt.

$124 \mathrm{Vgl}$. Gattorna/Walters (1996), S. 12, ,supply chain management is the preferred name for the actualization of ,integrated logistics' theory“", Bechtel/Jayaram (1997), S. 16; Heusler (2003), S. 167; Kaluza/Blecker (2000), S. 123-125.

125 Vgl. bspw. Dobler/Starling (2002), S. 96; Kuhn/Hellingrath (2002), S. 10; Schinzer (1999), S. 858, Hahn (2000), S. 12 ff. sowie Werner (2000), S. 815.

$126 \mathrm{Vgl}$. Larisch (1999), S. $135 \mathrm{ff}$. 
chen Wertschöpfungsnetzwerkes ist bzw. sind, ermöglicht einen Supply Chainorientierten Zugang zur Analyse der Leistungs-, Informations- und Finanzmittelflüsse.

„Kundenwunsch“ in diesem Sinne ist das Interesse der Gemeinschaft bzw. seiner Bürger an Leistungen, die (nur) ein öffentliches Gemeinwesen erbringt bzw. erbringen kann. Darunter fallen klassische öffentliche Güter wie äußere und innere Sicherheit. ${ }^{127}$ Dieser Kundenwunsch kann wegen der Besonderheiten öffentlicher Güter nicht direkt über Zahlungsbereitschaften geäußert werden, sondern artikuliert sich gemäß dem Demokratieprinzip über gewählte Volksvertreter (Analyseebene Netzwerk der Politik). Zahlungsströme erfolgen dann indirekt über Steuern bzw. Abgaben. Die damit erstellten Leistungen schlagen sich im individuellen Nutzenkalkül der Bürger nieder, bei denen bspw. der Bedarf nach (äußerem) Frieden gedeckt wird.

Welche Leistungen bzw. öffentlichen Aufgaben von einer Public Supply Chain erbracht werden, hängt damit sehr wohl von der insgesamten Zahlungsbereitschaft der Bürger ab. Sollte die Bereitschaft der Bürger zur Zahlung von Steuern dauerhaft und mehrheitlich zurückgehen, weil die politische Legitimierung vernachlässigt wird, so sind Public Supply Chains in ihrer Existenz dauerhaft bedroht. Public Supply Chain Management kann eine derartige Frage aus rein betriebswirtschaftlicher Perspektive nicht lösen, durch Einbeziehung politikwissenschaftlicher Erkenntnisse aber möglicherweise schon.

Konzeptionsmerkmal (4): Prozessorientierung

Während die Flussorientierung ein konstituierendes Merkmal für Supply Chains ist, umfasst die Prozessorientierung explizite Gestaltungsaspekte des Management einer Supply Chain. ${ }^{128}$ Im Sinne der oben skizzierten Rückkopplung zwischen politischen und ökonomischen Prozessen ist dabei für das Public SCM insbesondere die Unterscheidung zwischen der Gestaltung des reinen Auftragsabwicklungsprozesses (inklusive der damit verbundenen Führungsaufgaben, sogenannte Auftragsmanagement) ${ }^{129}$ und der weiterführenden Integration vorgelagerter Struktur- und Entwicklungsprozesse inklusive Ressourcengestaltung relevant. ${ }^{130}$ Auftragsabwicklung und Auftragsmanagement für öffentliche Aufgaben würden sich weitgehend an den genannten Effizienzzielen orientieren; Struktur-, Entwicklungs- und Ressourcenprozessmanagement berücksichtigt explizit auch Effektivitätsziele. Damit sind aber zugleich „Business Processes“ ${ }^{\text {“131 }}$ und Prozesse auf der Ebene der politischen Netzwerke involviert. Aufgabe eines ökonomisch-betriebswirtschaftlichen Public Supply Chain Management wäre deren Analyse auf Basis der Neuen Politischen Ökonomik. ${ }^{132}$

Konzeptmerkmal (5): Managementorientierung

Der mit der Managementorientierung verbundene Gestaltungsanspruch orientiert sich einerseits an institutionellen Merkmalen des Management. In privatwirtschaftlichen

127 Vgl. zu öffentlichen Gütern Budäus/Grüning (1997), S. 28 ff.

128 Vgl. Corsten (2002), S. 950; Hagen/Springer/Stabenau (2002), S. 11 und S. 20 sowie Kuhn/Hellingrath (2002), S. 27-29.

$129 \mathrm{Vgl}$. Specht/Hellmich (2000), S. 98 f.

130 Vgl. Bechtel/Jayaram (1997), S. 25; Hahn (2000), S. 14; Lambert/Cooper (2000), S. 72 ff.; Schönsleben/Hieber (2002), S. 47 und S. 56 sowie Specht/Hellmich (2000), S. 99 ff.

131 Cooper/Lambert/Pagh (1997), S. 2.

132 Vgl. zur neuen politischen Ökonomie Kyrer (2001). 
Supply Chains manifestiert sich dies in Führungsstrukturen für Supply Chains wie Supply Chain-Committees oder Supply Chain-Projektausschüssen zur unternehmensübergreifenden Koordination. ${ }^{133}$ Diese Strukturen sind prinzipiell auch auf Public Supply Chains zu übertragen; eine normative Führung erfolgt jedoch zumindest bislang über politische Institutionen im Netzwerk, welche den Bürger- bzw. Kundenwillen repräsentieren (sollen). Die Führungsstruktur des öffentlichen Sektors lässt sich weitergehend am funktionalen Supply Chain-Managementverständnis erklären. Es definiert eindeutige Gestaltungselemente wie (strategische) Planung, Durchführung, Kontrolle des öffentlichen Wertschöpfungsnetzwerkes und sichert so den weitergehenden Anspruch eines (Private und Public) SCM als ,integrationsorientierte Führungslehre“134 bzw. als „Management Philosophy ${ }^{6135}$ ab. ${ }^{136}$ Das bedeutet zwangsläufig, dass das Public SCM Entscheidungen einzelner Institutionen überlagern muss - Corsten (2002) spricht von einem „Eingriff in die ... Autonomie der Beteiligten. “137 Die netzwerkübergreifende Sichtweise erfordert eine Darstellung der Führungsebenen für eine gesamtheitliche Koordination der Public SC. Die Netzwerkebene der Politik wird im organisatorischen Sinn als Spitze der vollziehenden Gewalt (Exekutive) durch die Regierung repräsentiert. ${ }^{138}$ Auf derselben Ebene dienen Parlamente (Legislative) zur „Kontrolle und Kritik“ der Regierungstätigkeit. Regierung und Parlament kommen im Public SCM die Rolle des strategischen Planers zu, was bspw. die Beratung über längerfristige Wirkungen (Outcome des Mehrebenensystems) und strategischer Zielsetzungen staatlicher Maßnahmen und Gesetze beinhaltet. ${ }^{139}$ Thom und Ritz sehen die ,neue Hauptfunktion der politischen Steuerung“ in der „Strategiefindung zur längerfristigen Staatsführung unter der Rollenteilung von Legislative und Exekutive. ${ }^{\text {"140 }}$ Ein Mangel der strategischen politischen Zielsetzung und Planung ist im „Denken in Wahlzyklen“ ${ }^{\mathrm{zu}}$ sehen. ${ }^{141}$ Insbesondere mit fortgeschrittenem Wahlzyklus werden lang-, wenn nicht selbst mittelfristige Planungen unmöglich. ${ }^{142}$ Nicht nur auf Regierungsebene stellen langfristige Planungen die Ausnahme dar, auch die administrative (Verwaltungs-) Ebene denkt im Rahmen von Budgetverhandlungen jahreszyklisch. ${ }^{143}$ Der von politischer Ebene intendierte Outcome wird hierbei operational anhand von Budgets definiert. ${ }^{144}$ Die Verwaltungen stellen die unteren Ebenen der Exekutive dar und haben die konkrete Umsetzung der von der politischen Netzwerkebene verabschiedeten Maßnahmen und Gesetze zur Aufgabe. Der administrativen Netzwerkebene

133 Vgl. Hahn (2000), S. 16.

134 Vgl. Kotzab (2000), S. 39.

135 Vgl. Mentzer u.a. (2001), S. 9 f.

136 Vgl. die Analogie im Private SCM bei Bechtel/Jayaram (1997), S. 20 ff.; Buscher (1999), S. 454 f.; Chopra/Meindl (2001), S. 6 f. und S. 28 ff.; Cooper/Lambert/Pagh (1997), S. 6 ff.; Corsten/Gössinger (2001), S. 151 ff.; Kuhn/Hellingrath (2002), S. 13 f.; Otto/Kotzab (2002), S. 143 ff.; Specht/Hellmich (2000), S. 99 ff.; Tan (2001), S. 44 und Wildemann (2000), S. 78.

137 Corsten (2002), S. 947.

138 Vgl. Grünenfelder, (1997), S. 7.

$139 \mathrm{Vgl}$. Thom/Ritz (2000), S. 72.

140 Thom/Ritz (2000), S. 72.

$141 \mathrm{Vgl}$. Grünenfelder (1997), S. 44.

$142 \mathrm{Vgl}$. Thom/Ritz (2000), S. 77.

$143 \mathrm{Vgl}$. Thom/Ritz (2000), S. 77.

144 Vgl. Grünenfelder (1997), S. 56. 
obliegt dabei die Ergebnisverantwortung über die Erreichung der mit der politischen Ebene vereinbarten Outputs innerhalb vorgegebener Budgetrestriktionen. ${ }^{145}$ Die Verwaltungsebene fungiert also als Schnittstelle zwischen dem öffentlichen Sektor und der Netzwerkebene der privatwirtschaftlicher Unternehmen, wobei es im Rahmen der Gewährleistungsverantwortung für die Erreichung des vereinbarten Outputs (Leistungsvereinbarung) unerheblich ist, ob die Durchführung durch Handlungen der Verwaltung und/oder durch Leistungen kommerzieller (privater) Anbieter erfolgt. Eine Übergewichtung kurzfristig wirksamer Maßnahmen findet sich eingeschränkt auch im privaten Sektor wieder. Kritiker der marktwertorientierten Unternehmenssteuerung (Shareholder Value) sehen eine Verzerrung des rechnerischen Shareholder Value durch kurzfristige Manipulationen zur positiven Beeinflussung der Quartalsberichte. ${ }^{146}$ So reagiert der Markt aufgrund seiner Informationsineffizienz auf Informationen, die sich später als falsch bzw. irrelevant herausstellen. ${ }^{147}$

Die im Rahmen unserer Analyse identifizierten Konzeptmerkmale zur Wertschöpfung in Netzwerken der öffentlichen Hand finden in Teilaspekten auch bislang bereits Beachtung - allerdings fehlt die integrierende „Klammer“ des Public SCM-Ansatzes. Tabelle 1 gibt einen ersten Überblick über ausgewählte bestehende Teilkonzepte und deren Einordnung in ein umfassendes Public SCM.

\begin{tabular}{|c|c|c|c|c|c|}
\hline & $\begin{array}{l}\text { Analyse- } \\
\text { objekt }\end{array}$ & Konzept & Fokus & $\begin{array}{l}\text { Aufgabenteilung/ } \\
\text { Mechanismen }\end{array}$ & $\begin{array}{l}\text { Einordnung in } \\
\text { Konzept- } \\
\text { merkmale } \\
\text { des Public SCM }\end{array}$ \\
\hline $\begin{array}{l}\text { Thom/ } \\
\text { Ritz } \\
\text { (2000) }\end{array}$ & $\begin{array}{l}\text { Führungs- } \\
\text { konzept für } \\
\text { den öffentli- } \\
\text { chen Sektor }\end{array}$ & $\begin{array}{l}\text { Konzept- } \\
\text { bausteine In- } \\
\text { novations- } \\
\text { management, } \\
\text { organisator. } \\
\text { Gestaltung und } \\
\text { Personal- } \\
\text { management }\end{array}$ & $\begin{array}{l}\text { Verschiedene } \\
\text { Steuerungs- } \\
\text { ebenen im } \\
\text { öffentlichen } \\
\text { Sektor, politi- } \\
\text { sche und be- } \\
\text { triebliche Steu- } \\
\text { erungsebene }\end{array}$ & $\begin{array}{l}\text { Definition von Kernaufgaben } \\
\text { der politischen Führung im } \\
\text { NPM, konkrete Umsetzung } \\
\text { auf der betrieblichen Ebene } \\
\text { (alle Institutionen des öffentli- } \\
\text { chen Sektors) und Definition } \\
\text { deren Verantwortungsbereiche }\end{array}$ & $\begin{array}{l}\text { (1) Netzwerk- } \\
\text { orientierung } \\
\text { (5) Management- } \\
\text { orientierung }\end{array}$ \\
\hline $\begin{array}{l}\text { Thom/ } \\
\text { Ritz } \\
\text { (2003) }\end{array}$ & $\begin{array}{l}\text { Wert- } \\
\text { schöpfungs- } \\
\text { kette einer } \\
\text { öffentlichen } \\
\text { Institution }\end{array}$ & $\begin{array}{l}\text { Integrativer } \\
\text { Führungs- } \\
\text { ansatz, öffent- } \\
\text { liche Wert- } \\
\text { schöpfungs- } \\
\text { kette als Sys- } \\
\text { tem aus gesell- } \\
\text { schaftlichen, } \\
\text { politischen, } \\
\text { ökonomischen } \\
\text { und techni- } \\
\text { schen Aktivitä- } \\
\text { ten }\end{array}$ & $\begin{array}{l}\text { Führung- und } \\
\text { Steuerung } \\
\text { öffentlicher } \\
\text { Wertschöpfung } \\
\text { auf verschiede- } \\
\text { nen Ebenen des } \\
\text { öffentlichen } \\
\text { Sektors, Einbe- } \\
\text { zug des priva- } \\
\text { ten Sektors zur } \\
\text { Leistungs- } \\
\text { erstellung }\end{array}$ & $\begin{array}{l}\text { Kombination politischer und } \\
\text { verwaltungsinterner Aktivitä- } \\
\text { ten, Problem des fehlenden } \\
\text { Preismechanismus, Unter- } \\
\text { scheidung in strategische Ziel- } \\
\text { bildung, Planung auf politi- } \\
\text { scher Ebene (Outcome, Im- } \\
\text { pact) und Strategieverantwor- } \\
\text { tung auf betrieblicher Ebene } \\
\text { (Output), Funktionsergänzung } \\
\text { durch komplementäre Aktivi- } \\
\text { täten und Ressourcen in PPPs }\end{array}$ & $\begin{array}{l}\text { (1) Netzwerk- } \\
\text { orientierung } \\
\text { (2) Zielorientie- } \\
\text { rung }\end{array}$ \\
\hline
\end{tabular}

145 Vgl. Thom/Ritz (2000), S. 73 und Kapitel 2.2.

146 Verpflichtung zur Quartalsberichterstattung, vgl. bspw. § 63 Börsenordnung der Frankfurter Wertpapierbörse. 147 Vgl. Franke/Hax (2004), S. 456 f., bspw. Mitarbeiterfreistellungen. 


\begin{tabular}{|c|c|c|c|c|c|}
\hline $\begin{array}{l}\text { Grünen- } \\
\text { felder } \\
\text { (1997) }\end{array}$ & $\begin{array}{l}\text { Verant- } \\
\text { wortungs- } \\
\text { Ketten- } \\
\text { Diagramm } \\
\text { der öffentli- } \\
\text { chen Hand }\end{array}$ & $\begin{array}{l}\text { Politische Füh- } \\
\text { rung im Rah- } \\
\text { men von NPM }\end{array}$ & \begin{tabular}{|l|} 
Führung, Steu- \\
erung und \\
Überwachung \\
auf unter- \\
schiedlichen \\
Ebenen der \\
offentlichen \\
Hand, Ausrich- \\
tung der Leis- \\
tung auf das \\
„Volk“
\end{tabular} & $\begin{array}{l}\text { Informationsanalyse und stra- } \\
\text { tegische Zielbildung als Auf- } \\
\text { gabe der Exekutivspitze, Wir- } \\
\text { kungsfestlegung (Outcome), } \\
\text { Ausrichtung an Stakeholderin- } \\
\text { teressen, Planung auf Parla- } \\
\text { ments- und Verwaltungsebene, } \\
\text { Umsetzung definierter Out- } \\
\text { comes in konkreten Outputs, } \\
\text { Planzustimmungsgewalt bei } \\
\text { der Legislative, Verwaltungs- } \\
\text { stellen als Leistungserbringer }\end{array}$ & $\begin{array}{l}\text { (1) Netzwerk- } \\
\text { orientierung } \\
\text { (5) Management- } \\
\text { orientierung }\end{array}$ \\
\hline $\begin{array}{l}\text { Borins/ } \\
\text { Grüning } \\
\text { (1998) }\end{array}$ & \begin{tabular}{|l} 
Bereit- \\
stellung \\
öffentlicher \\
Güter
\end{tabular} & $\begin{array}{l}\text { Alternativen } \\
\text { zum traditio- } \\
\text { nellen öffentli- } \\
\text { chen Monopol } \\
\text { durch „markt- } \\
\text { ähnliche Me- } \\
\text { chanismen“" }\end{array}$ & \begin{tabular}{|l|} 
Führung und \\
Steuerung der \\
öffentlichen \\
Hand durch \\
Wettbewerb, \\
Preise, monetä- \\
re Anreize usw.
\end{tabular} & $\begin{array}{l}\text { Vermehrte Einbeziehung des } \\
\text { privaten Sektors, Steuerung } \\
\text { durch (verwaltungs-) interne } \\
\text { Preise und Märkte, Marktme- } \\
\text { chanismus als Anreizstruktur } \\
\text { (Public Choice) }\end{array}$ & $\begin{array}{l}\text { (5) Management- } \\
\text { orientierung }\end{array}$ \\
\hline $\begin{array}{l}\text { Naschold } \\
\text { u.a. } \\
\text { (2000) }\end{array}$ & $\begin{array}{l}\text { Wert- } \\
\text { schöpfungs- } \\
\text { prozess des } \\
\text { öffentlichen } \\
\text { Sektors }\end{array}$ & $\begin{array}{l}\text { Wertschöpfung } \\
\text { der öffentli- } \\
\text { chen Hand als } \\
\text { verwaltungs- } \\
\text { und unterneh- } \\
\text { mens- } \\
\text { übergreifender } \\
\text { Leistungs- } \\
\text { zusammenhang } \\
\text { (mehrerer pri- } \\
\text { vater und öf- } \\
\text { fentlicher Ak- } \\
\text { teure) }\end{array}$ & \begin{tabular}{|l|} 
Gestaltung der \\
Leistungskette \\
(Leistungstiefe, \\
Kooperations- \\
formen usw.), \\
Nutzenzuwachs \\
des Bürgers
\end{tabular} & $\begin{array}{l}\text { Gestufte Analyse der Aufga- } \\
\text { benteilung öffentlich-privat, } \\
\text { (1) Konzept der strategischen } \\
\text { Relevanz der (öffentlichen) } \\
\text { Leistung, politische Strategie- } \\
\text { und Zielbildung (Definition } \\
\text { von Zielen, Impact und Output } \\
\text { und Outcome auf unterschied- } \\
\text { lichen Ebenen des öffentlichen } \\
\text { Sektors), politische Steuerung } \\
\text { (Steuerbarkeit politisch ge- } \\
\text { wollter Leistungsmerkmale, } \\
\text { Gewährleistung), (2) transak- } \\
\text { tionskosten-ökonomische } \\
\text { Analyse }\end{array}$ & $\begin{array}{l}\text { (1) Netzwerk- } \\
\text { orientierung }\end{array}$ \\
\hline $\begin{array}{l}\text { Erridge/ } \\
\text { McIlroy } \\
\text { (2003) }\end{array}$ & $\begin{array}{l}\text { Liefer- } \\
\text { zusammen- } \\
\text { hänge des } \\
\text { öffentlichen } \\
\text { Sektors als } \\
\text { Netzwerk } \\
\text { vom Liefe- } \\
\text { ranten bis } \\
\text { zum End- } \\
\text { verbraucher } \\
\text { (Bezie- } \\
\text { hungs- } \\
\text { geflecht) }\end{array}$ & $\begin{array}{l}\text { Konkurrierende } \\
\text { Typen der } \\
\text { öffentlichen } \\
\text { Beschaffung } \\
\text { (reguliert, } \\
\text { sozial) und des } \\
\text { SCM des priva- } \\
\text { ten Sektors } \\
\text { (kommerziell) }\end{array}$ & \begin{tabular}{|l|} 
Einführung von \\
betriebswirt- \\
schaftlichen \\
Führungs- und \\
Steuerungs- \\
mechanismen \\
im öffentlichen \\
Sektor
\end{tabular} & $\begin{array}{l}\text { Besondere Sachzwänge des } \\
\text { öffentlichen Sektors, auch bei } \\
\text { Fremdvergabe verbleibt Ver- } \\
\text { antwortung gegenüber der } \\
\text { Öffentlichkeit, Privatisierung } \\
\text { und öffentlich-private Partner- } \\
\text { schaften führen zu einer An- } \\
\text { wendung des kommerziellen } \\
\text { Typs in der öffentlichen Be- } \\
\text { schaffung, Modell zur Defini- } \\
\text { tion von Beschaffungsstrate- } \\
\text { gien, ansatzweiser Einschluss } \\
\text { von Finanz- und Informations- } \\
\text { flüssen }\end{array}$ & $\begin{array}{l}\text { (1) Netzwerk- } \\
\text { orientierung } \\
\text { (2) Ziel- } \\
\text { orientierung } \\
\text { (3) Fluss- } \\
\text { orientierung }\end{array}$ \\
\hline
\end{tabular}

Tab. 1: Bestehende Teilkonzepte eines Public SCM

Quelle: Eigene Darstellung 
Die an einer Public Supply Chain beteiligten politischen, administrativen und/oder kommerziellen Institutionen müssen keineswegs partnerschaftlich verflochten sein. Mag die Literatur auch überwiegend von einer Intra-Chain-Kooperation ausgehen, ${ }^{148}$ tatsächlich haben Cooper, Lambert und Pagh bzw. Lambert und Cooper für privatwirtschaftliche Supply Chains zu Recht darauf hingewiesen, dass SCM durchaus auch bedeuten kann, einzelne Beziehungen innerhalb einer Supply Chain keineswegs aktiv-kooperativ gestalten zu müssen. ${ }^{149}$ So genannte „Not-Managed Process Links“ werden bewusst nicht in ein aktives Management eingebunden, da sie bspw. keinerlei strategische Bedeutung für den im Sinne der oben genannten Zielorientierung gemessenen Erfolg einer Supply Chain haben. ${ }^{150}$ Exemplarisch sei das Wettbewerbspostulat des öffentlichen Vergaberechts genannt, welches mit seinen Competitive Tendering-Verfahren ja ebenfalls keine langfristige Lieferantenbeziehung vorsieht. Derselben Argumentation folgen Erridge und McIlroy. Sie unterscheiden zur Analyse von Aufträgen im öffentlichen Sektor „Competitive Tendering“, „Supply Management“ sowie „Supply Chain Co-ordination Networks“. Als drei wesentliche Strukturtypen reicht deren Ausgestaltung von einer rein marktlich wettbewerblichen bis hin zu einer stark partnerschaftlich ausgerichteten Zusammenarbeit. ${ }^{151}$

Die Unterscheidung zwischen kurzfristig ausgerichteten, bilateralen Abnehmer-ZulieferBeziehungen zur Erfüllung öffentlicher Ausgaben und der langfristig angelegten Gestaltung eines öffentlichen Wertschöpfungsnetzwerks bestimmt auch die Differenzierung von Public SCM im weiteren Sinne und Public SCM im engeren Sinne (vgl. Abbildung 5). Während sich Public Supply Chain Management im engeren Sinne tatsächlich auf intensive, mindestens trilaterale Netzwerke politischer, administrativer und kommerzieller Institutionen zur öffentlichen Leistungserstellung konzentriert, umfasst Public Supply Chain Management im weiteren Sinne auch die Beziehungen, die bspw. im klassischen Vergabeverfahren an einen privaten Lieferanten vergeben werden. Alle diese Institutionen tragen zur öffentlichen Wertschöpfung bei.

148 Vgl. Arnold/Warzog (2001), S. 23 f.; Hahn (2000), S. 13; ähnlich bei Burt/Starling (2002), S. 97; Kaluza/Blecker (2000), S. 123 und S. 127; Kuhn/Hellingrath (2002), S. 23-27; Otto/Kotzab (2002), S. 142 f.; Pinkerton (2002), S. 517; Specht/Hellmich (2000), S. 92 f. und Zimmermann (2003), S. 36 ff.

149 Vgl. Cooper/Lambert/Pagh (1997), S. 3 und S. 9 sowie Lambert/Cooper (2000), S. 69 f. und S. 74 ff.

150 Vgl. Lambert/Cooper (2000), S. 75 und die ähnliche Argumentation bei Klaus (1999), S. 128 zur „Festigkeitsdimension (looseness/tightness)“ sowie bei Tan (2001), S. 40, „Technically, the value chain is too complex to achieve a full integration of all ... entities within it in order to reap the benefits offered by supply chain management"

151 Vgl. Erridge/McIlroy (2003), S. 561. 


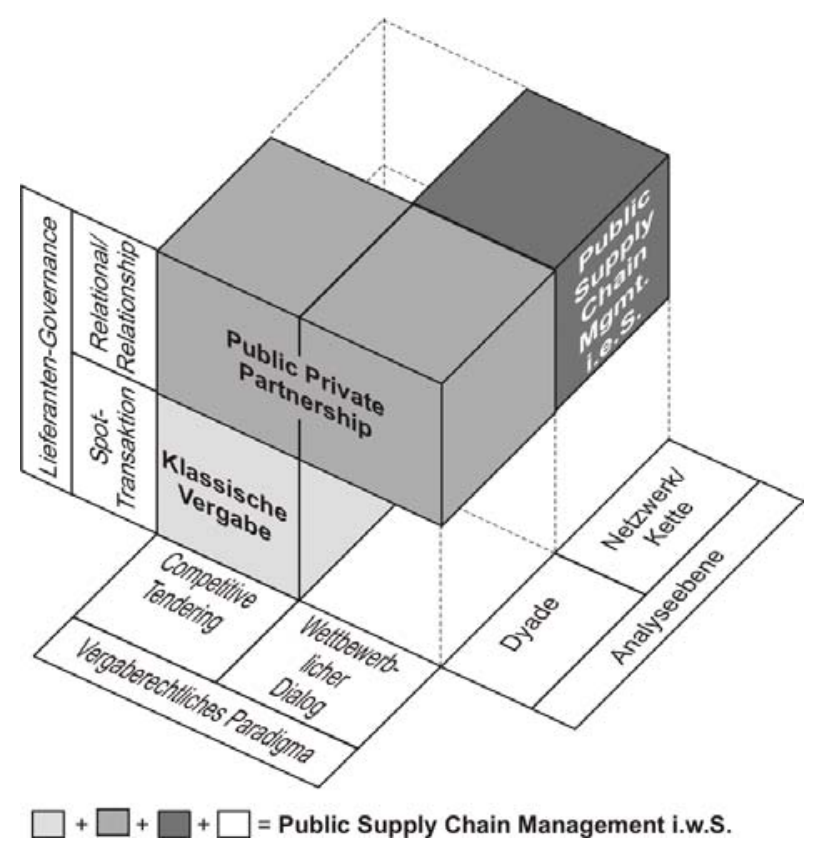

Abb. 5: Abgrenzung des Public Supply Chain Management zu verwandten Konzepten Quelle: Eigene Darstellung

Der dargestellte konzeptionelle Ansatz des Public Supply Chain Management gewährleistet eine umfassende Systematisierung mit einem Anspruch auf generelle Gültigkeit. ${ }^{152}$ Für einen konkreten Handlungsbezug und Instrumenteneinsatz sind aufgrund der Komplexität Teilprobleme zu extrahieren und Ansätze des Private SCM in gleicher Weise zu prüfen.

\section{Abstract}

Michael Eßig and Alexander Batran, A Conceptual Framework for Public Supply Chain Management

Added Value; Multi-Level-Network; Network; Public Procurement; Public Supply Chain; Supply Chain Management

In private sector research supply chain management is an often and extensive discussed topic. We used these findings, considering a continuing implementation of market mechanisms in the public sector, to develop a conceptual framework for public supply chain management. Not only legal aspects are implied, rather a multi-level-network, consisting of politics, administration, and additionally private sector companies must be examined explicitly. 


\section{Literaturverzeichnis}

Adams, Michael (2004), Ökonomische Theorie des Rechts - Konzepte und Anwendungen, 2. Aufl., Frankfurt am Main u.a.

Adler, Jost (1996), Informationsökonomische Fundierung von Austauschprozessen, Trier und Wiesbaden

Arnold, Ulli (1993), Wertschöpfungspartnerschaften als Erfolgspotentiale, in: Beschaffung Aktuell, o. Jg., Nr. 10, S. $20-25$

Arnold, Ulli, u.a. (2005), Thesen zum Entwicklungsstand einer dynamischen Disziplin, in: Controlling, 17. Jg., Nr. 1, S. 41-48

Arnold, Ulli (1997), Beschaffungsmanagement, 2. Aufl., Stuttgart

Arnold, Ulli (2004), Ökonomisierung der Streitkräfte als Gestaltungsproblem der Inneren Führung, in: Gesellschaft, Militär, Krieg und Frieden im Denken von Wolf Graf von Baudissin, hrsg. von Martin Kutz, Baden Baden, S. $143-154$

Arnold, Ulli und Frank Warzog (2001), Supply Chain Management - Konzeptabgrenzung und branchenspezifische Differenzierung, in: Supply Chain Management: Unternehmensübergreifende Prozesse, Kollaboration, ITStandards, hrsg. von Ulli Arnold, Reinhold Mayer und Georg Urban, Bonn, S. 13-47

Backhaus, Klaus (2003), Industriegütermarketing, 7. Aufl., München 2003

Bartelt, Andreas (2002), Vertrauen in Zuliefernetzwerken, Wiesbaden

Batran, Alexander, Michael Eßig und Berthold Schäfer (2004), Public-Private Partnerships as an Element of Public Procurement Reform in Germany, in: Challenges in Public Procurement - An International Perspective, hrsg. von Khi V. Thai u.a., S. 127-149

Bayón, Tomás (1997), Neuere Mikroökonomie und Marketing - Eine wissenschaftstheoretisch geleitete Analyse, Wiesbaden

Bea, Franz X., Birgit Friedl und Marcell Schweitzer (2004), Allgemeine Betriebswirtschaftslehre, Band 1, 9. Aufl., Stuttgart

Bechtel, Christian und Jayanth Jayaram (1997), Supply Chain Management, A Strategic Perspective, in: International Journal of Logistics Management, 8. Jg., Nr. 1, S. 15-34

Bundesverband für Materialwirtschaft, Einkauf und Logistik e.V. (BME) und Booz, Allen \& Hamilton (2000), Chancen und Entwicklungen im Public Procurement, Studie, Berlin 2000

Bogaschewsky, Ronald (1994), Rationalisierungsgemeinschaften mit Lieferanten, in: Konzernlogistik und Rationalisierungsgemeinschaften mit Lieferanten, hrsg. von Jürgen Bloech, Ronald Bogaschewsky und Werner Frank, Stuttgart, S. 95-115

Bogaschewsky, Ronald (2003), Historische Entwicklung des Beschaffungsmanagements, in: Management und Controlling von Einkauf und Logistik, Festschrift für Jürgen Bloech, hrsg. von Ronald Bogaschewsky und Uwe Götze, Gernsbach, S. 13-42

Borins, Sandford und Gernod Grüning (1998), New Public Management - Theoretische Grundlagen und problematische Aspekte der Kritik, in: New Public Management, hrsg. von Dietrich Budäus, Peter Conrad und Georg Schreyögg, Berlin und New York, S. 11-53

Bozdogan, Kikor u.a. (1998), Architectural innovation in product development through early supplier integration, in: R\&D Management, 28. Jg. Nr. 3, S. 163-173

Brümmerhoff, Dieter (2001), Finanzwissenschaft, 8. Aufl., Oldenbourg

Budäus, Dietrich (1988), Einzelwirtschaftliche Effizienzanalyse privater und öffentlicher Leistungserstellung in der Privatisierungsdiskussion, in: Privatisierung und die Zukunft der öffentlichen Wirtschaft, hrsg. von Helmut Brede, Baden Baden, S. 203-222

Budäus, Dietrich (1994), Public Management - Konzepte und Verfahren zur Modernisierung öffentlicher Verwaltungen, Berlin

Budäus, Dietrich (1998), Von der bürokratischen Steuerung zum New Public Management - Eine Einführung, in: New Public Management, hrsg. von Dietrich Budäus, Peter Conrad und Georg Schreyögg, Berlin und New York, S. 1-10

Budäus, Dietrich (2003), Neue Kooperationsformen zur Erfüllung öffentlicher Aufgaben - Charakterisierung, Funktionsweise und Systematisierung von Public Private Partnership, in: Die Ökonomisierung des öffentlichen Sektors - Instrumente und Trends, hrsg. von Jens Harms und Christoph Reichard, Baden Baden, S. 213-233

Budäus, Dietrich und Gernod Grüning (1997), Public Private Partnership - Konzeption und Probleme eines Instruments zur Verwaltungsreform aus Sicht der Public Choice Theorie, in: Public Private Partnership - Neue Formen öffentlicher Aufgabenerfüllung, hrsg. von Dietrich Budäus und Peter Eichhorn, Baden Baden, S. 25-66

Bundesministerium für Wirtschaft und Arbeit (30. Oktober 2004), Begründung des Gesetzes zur Neuregelung des Vergaberechts, http://www.bmwa.bund.de/Redaktion/Inhalte/Pdf/arbeits-entwurf-des-bmwa-begruendungdesgesetzeszurneurgelung-des-vergaberechts,property=pdf.pdf

Burt, David N., Donald W Dobler und Stephen L. Starling (2003), World Class Supply Management - The Key to Supply Chain Management, 7. Aufl., New York 
Busch, Axel und Wilhelm Dangelmaier (2002), Integriertes Supply Chain Management - Ein koordinationsorientierter Überblick, in: Integriertes Supply Chain Management - Theorie und Praxis effektiver unternehmensübergreifender Geschäftsprozesse, hrsg. von Axel Busch und Wilhelm Dangelmaier, Wiesbaden, S. 1-21

Buscher, Udo (1999), ZP-Stichwort - Supply Chain Management, in: Zeitschrift für Planung, 10. Jg., o. Nr., S. 449456

Coase, Ronald H. (1937), The Nature of the Firm, in: Economica, 4. Jg., o. Nr., S. 386-405

Cooper, Martha C., Douglas M. Lambert und Janus D. Pagh (1997), Supply Chain Management - More Than a New Name for Logistics, in: International Journal of Logistics Management, 8. Jg., Nr. 1, S. 1-14

Chopra, Sunil und Peter Meindl (2001), Supply Chain Management - Strategy, Planning and Operation, Upper Saddle River

Corsten, Hans (2002), Herausforderungen an das Supply Chain Management im internationalen Unternehmensverbund, in: Handbuch Internationales Management, 2. Aufl., hrsg. von Klaus Macharzina und Michael-Jörg Oesterle, Wiesbaden, S. 943-968

Corsten, Hans und Ralf Gössinger (2001), Einführung in das Supply Chain Management, München und Wien

Darby, Michael R. und Edi Karni (1973), Free Competition and the Optimal Amount of Fraud, in: Journal of Law and Economics, 16. Jg., Nr. 4, 67-88

Ebers, Mark und Wilfried Gotsch (2002), Institutionenökonomische Theorien der Organisation, in: Organisationstheorien, 5. Aufl., hrsg. von Alfred Kieser, Stuttgart, S. 199-251

Eichhorn, Peter (1987), Was können Unternehmen und Verwaltungen voneinander lernen?, in: Baden-Württembergische Verwaltungspraxis, 14. Jg., Nr. 10, S. 217-221

Eichhorn, Peter (1995), Public Private Partnership - Praxis, Probleme, Perspektiven, in: Sozialpolitik und öffentliche Wirtschaft, hrsg. von Lothar F. Neumann und Frank Schulz-Nieswandt, Berlin, S. 173-184

Eichhorn, Peter (2001), Öffentliche Betriebswirtschaftslehre als eine Spezielle BWL, in: WiSt, 30. Jg., Nr. 2, S. 409416

Eisenbarth, Marc (2003), Erfolgsfaktoren des Supply Chain Managements in der Automobilindustrie, Frankfurt am Main

Engelhardt, Werner H., Michael Kleinaltenkamp und Martin Reckenfelderbäumer (1993), Leistungsbündel als Absatzobjekte - Ein Ansatz zur Überwindung der Dichotomie von Sach- und Dienstleistungen, in: ZfbF, 45. Jg., Nr. 5, S. 395-426

Erridge, Andrew und Jahn McIlroy (2003), Öffentliches Beschaffungswesen und Steuerung der Beschaffung, in: Handbuch Beschaffung, hrsg. von Roman Boutellier, Stephan M. Wagner und Hans P. Wehrli, München und Wien, S. 547-570

Eßig, Michael (2003), Supplier Lifetime Value als Ansatz zur Neubewertung von Lieferantenbeziehungen, in: Integrated Supply Management - Einkauf und Beschaffung: Effizienz steigern, Kosten senken, hrsg. von Ronald Bogaschewsky, Köln, S. 323-346

Eßig, Michael (2005), Integriertes Beschaffungsmanagement - Der Ansatz des „House of Sourcing and Supply Management“, in: Perspektiven des Supply Management: Konzepte und Anwendungen, hrsg. von Michael Eßig, Berlin, Heidelberg und New York, S. 3-26

Fante, Jan (2004), Die Instrumentalisierung des öffentlichen Beschaffungswesen zur Durchsetzung politischer Ziele, Aachen

Franke, Günther und Herbert Hax (2004), Finanzwirtschaft des Unternehmens und Kapitalmarkt, 5. Aufl., Berlin, Heidelberg und New York

Gattorna, John L. und D. W. Walters (1996), Managing the Supply Chain - A Strategic Perspective, Houndmills

Göbel, Elisabeth (2002), Neue Institutionenökonomik - Konzeption und betriebswirtschaftliche Anwendungen, Stuttgart

Göbel, Elisabeth (2004), Neue Institutionenökonomik und ihre mögliche Bedeutung für die Organisation der Wahrnehmung öffentlicher Aufgaben, in: Neue Institutionenökonomik, Public Private Partnerships, Gewährleistungsstaat, Tagungspapier des Wissenschaftlichen Beirates der Gesellschaft für öffentliche Wirtschaft, hrsg. von Elisabeth Göbel u.a., Berlin, S. 3-21

Göpfert, Ingrid (2002), Einführung, Abgrenzung und Weiterentwicklung des Supply Chain Managements, in: Integriertes Supply Chain Management - Theorie und Praxis unternehmensübergreifender Geschäftsprozesse, hrsg. von Axel Busch und Wilhelm Dangelmaier, Wiesbaden, S. 25-44

Greiling, Dorothea (1994), Neuere Formen von Public Private Partnerships, in: Akademie, 19. Jg., Nr. 3, S. $72-75$

Grünenfelder, Peter (1997), Die Rolle der politischen Führung im New Public Management, Bern, Stuttgart und Wien

Hagen, Nils, Verena Springer und Hanspeter Stabenau (2002), Gestaltungsfeld Prozessmanagement, in: Management integrierter logistischer Netzwerke, hrsg. von Helmut Baumgarten u.a., Bern u.a., S. 9-104

Hahn, Dietger (2000), Problemfelder des Supply Chain Management, in: Supply Chain Management, hrsg. von Horst Wildemann, München, S. 9-19

Halldorsson, Arni, Tage Skjott-Larsen und Herbert Kotzab (2003), Interorganizational Theories Behind Supply Chain Management - Discussion and Applications, in: Strategy and Organization in Supply Chains, hrsg. von Stefan Seuring u.a., Heidelberg, S. 165-180 
Hammann, Peter (2004), Öffentliche und private Unternehmen als Institution zur Erbringung von Diensten von und für Menschen, in: Zeitschrift für öffentliche und gemeinwirtschaftliche Unternehmen, 27. Jg., Nr. 1, S. 87-95

Hammerschmid, Gerhard (2001), New-public-Management zwischen Konvergenz und Divergenz - Eine institutionentheoretische Betrachtung anhand dreier Fallstudien der internationalen Verwaltungspraxis im Politikfeld öffentlicher Personennahverkehr, Wien

Harding, Michael und Mary Lu Harding (1991), Purchasing, New York

Harms, Jens und Christoph Reichard (2003), Ökonomisierung des öffentlichen Sektors - Eine Einführung, in: Die Ökonomisierung des öffentlichen Sektors - Instrumente und Trends, hrsg. von Jens Harms und Christoph Reichard, Baden Baden, S. 13-17

Heusler, Klaus Felix (2003), Competencies in Supply Chain Management - The Contribution of the Resource-Based View of the Firm, in: Strategy and Organization in Supply Chains, hrsg. von Stefan Seuring u.a., Heidelberg, S. $165-180$

Kaluza, Bernd und Thorsten Blecker (2000), Supply Chain Management und Unternehmung ohne Grenzen - Zur Verknüpfung zweier interorganisationaler Konzepte, in: Supply Chain Management, hrsg. von Horst Wildemann, München, S. 117-152

Kantzenbach, Erhard (1967), Die Funktionsfähigkeit des Wettbewerbs, Wirtschaftspolitische Studien des Instituts für Europäische Wirtschaftspolitik der Universität Hamburg, 2. Aufl., Göttingen

Klaus, Peter (1999), Die organisatorische Integration von Versorgungsketten: „Best Practices“ für die Organisation des Supply Chain Managements in führenden US-amerikanischen Unternehmen, in: Logistikforschung - Entwicklungszüge und Gestaltungsperspektiven, hrsg. von Hans-Christian Pfohl, Berlin, S. 109-138

Kommission der europäischen Gemeinschaften (30. Oktober 2004), Grünbuch zu öffentlich-privaten Partnerschaften und den gemeinschaftlichen Rechtsvorschriften für öffentliche Aufträge und Konzessionen, http://europa.eu. int/eur-lex/de/com/gpr/2004/com2004_0327de01.pdf

Kotzab, Herbert (2000), Zum Wesen von Supply Chain Management vor dem Hintergrund der betriebswirtschaftlichen Logistikkonzeption - Erweiterte Überlegungen, in: Supply Chain Management, hrsg. von Horst Wildemann, München, S. 21-47

Kuhn, Axel und Bernd Hellingrath (2002), Supply Chain Management - Optimierte Zusammenarbeit in der Wertschöpfungskette, Berlin

Kyrer, Alfred (2001), Neue politische Ökonomie 2005, München und Wien

Lambert, Douglas M. und Martha C. Cooper (2000), Issues in Supply Chain Management, in: Industrial Marketing Management, 29. Jg., Nr. 1, S. 65-83

Lambert, Douglas M., Margaret A. Immelhainz und John T. Gardner (1996), Developing and implementing supply chain partnerships, in: The International Journal of Logistics Management, 7. Jg., Nr. 2, S.1-17

Larisch, Matthias (1999), Elemente einer Ökonomie sozialwirtschaftlicher Organisationen, Frankfurt am Main

Lenk, Thomas (2004), Neue Institutionenökonomik und ihre mögliche Bedeutung für die Organisation der Wahrnehmung öffentlicher Aufgaben, in: Neue Institutionenökonomik, Public Private Partnerships, Gewährleistungsstaat, Tagungspapier des Wissenschaftlichen Beirates der Gesellschaft für öffentliche Wirtschaft, hrsg. von Elisabeth Göbel u.a., Berlin, S. 22-33

Löffler, Elke (2003), Die Ökonomisierung des Staates - Versuch einer Begriffserklärung, in: Die Ökonomisierung des öffentlichen Sektors - Instrumente und Trends, hrsg. von Jens Harms und Christoph Reichard, BadenBaden, S. 19-26

Macneil, Ian R. (1978), Contracts - Adjustment of long-term-economic relations under classical, neoclassical, and relational contract law, in: Northwestern University Law Review, 72. Jg., o. Nr., S. 854-906

Mantzavinos, Chrysostomos (1994), Positive und normative Wettbewerbstheorie - der Versuch einer Systematisierung, in: Probleme der unvollkommenen Konkurrenz, hrsg. von Alfred E. Ott, Tübingen und Basel, S. 65-73

Marbacher, Albert (2001), Demand \& Supply Chain Management - Zentrale Aspekte der Gestaltung und Überwachung unternehmensübergreifender Leistungserstellungsprozesse betrachtet aus der Perspektive eines Markenartikelherstellers aus der Konsumgüterindustrie, Bern u.a.

Mentzer, John T. u.a. (2001), What is Supply Chain Management?, in: Supply Chain Management, hrsg. von John T. Mentzer, Thousand Oaks, S. 1-26

Möller, Klaus (2003), Supply Chain Valuation - Wertschöpfung in und durch Supply Chain Networks, in: Supply Chain Controlling in Theorie und Praxis - Aktuelle Konzepte und Unternehmensbeispiele, hrsg. von Wolfgang Stölzle und Andreas Otto, Wiesbaden, S. 49-82

Morgan, Robert M. und Shelby D. Hunt (1994), The Commitment-Trust Theory of Relationship Marketing, in: Journal of Marketing, 58. Jg., Nr. 3, S. 20-38

Mühlenkamp, Holger (2003), Zum grundlegenden Verständnis einer Ökonomisierung des öffentlichen Sektors - Die Sicht der Ökonomen, in: Die Ökonomisierung des öffentlichen Sektors - Instrumente und Trends, hrsg. von Jens Harms und Christoph Reichard, Baden Baden, S. 47-73

Müller Martin, Stefan Seuring und Maria Goldbach (2003), Supply Chain Management - Neues Konzept oder Modetrend?, in: Die Betriebswirtschaft, 63. Jg., Nr. 4, S. 419-439

Naschold, Frieder u.a. (2000), Leistungstiefe im öffentlichen Sektor, 2. Aufl., Berlin

Neher, Axel (2003), Wertorientierung im Supply Chain Controlling, in: Supply Chain Controlling in Theorie und Praxis, hrsg. von Wolfgang Stölzle und Andreas Otto (2003), S. 29-47 
Nelson, Phillips (1970), Information and Consumer Behavior, in: Journal of Political Economy, 78. Jg., Nr. 2, S. 311-329

Oberender, Peter (1995), Industrieökonomik, in: Allgemeine Wirtschaftstheorie, hrsg. von Norbert Berthold, München, S. 31-52

o. V. (2001), Vergaberecht, 4. Aufl., München

Otto, Andreas und Herbert Kotzab (2002), Ziel erreicht? Sechs Perspektiven zur Ermittlung des Erfolgsbeitrags des Supply Chain Management, in: Handbuch Industrielles Beschaffungsmanagement - Internationale Konzepte, innovative Instrumente, aktuelle Praxisbeispiele, 2. Aufl., hrsg. von Dietger Hahn und Lutz Kaufmann, Wiesbaden, S. $125-150$

Owens Swift, Cathy und Barbara J. Coe (1994), Sourcing Preference Scale, in: Industrial Marketing Management, 23. Jg., Nr. 2, S. 171-180

Parker, David und Keith Hartley (2003), Transaction costs, relational contracting and public private partnerships: a case study of UK defence, in: Journal of Purchasing \& Supply Management, 9. Jg., Nr. 3, S. 97-108

Pfohl, Hans-Christian (1999), Konzept des Supply Chain Managements und das Berufsbild des Logistikmanagers, in: Logistikforschung - Entwicklungszüge und Gestaltungsperspektiven, hrsg. von Hans-Christian Pfohl, Berlin, S. 173-228

Picot, Arnold (1991), Ein neuer Ansatz zur Gestaltung der Leistungstiefe, in: ZfbF, 43. Jg., Nr. 4, S. 336-357

Picot, Arnold, Helmut Dietl und Egon Franck (2002), Organisation - Eine ökonomische Perspektive, 3. Aufl., Stuttgart

Pinkerton, Richard L. (2002), The Evolution of Purchasing to Supply Chain Management, in: Handbuch Industrielles Beschaffungsmanagement - Internationale Konzepte, innovative Instrumente, aktuelle Praxisbeispiele, 2. Aufl., hrsg. von Dietger Hahn und Lutz Kaufmann, Wiesbaden, S. 507-522

Prahalad, C. K. und Gary Hamel (1990), The Core Competence of the Corporation, in: Harvard Business Review, 68. Jg., Nr. 3, S. 79-91

Präuer, Arndt (2004), Solutions Sourcing - Strategien und Strukturen interorganisationaler Wertschöpfungssysteme, Wiesbaden

Reichard, Christoph (2004), Das Konzept des Gewährleistungsstaates, in: Neue Institutionenökonomik, Public Private Partnerships, Gewährleistungsstaat, Tagungspapier des Wissenschaftlichen Beirates der Gesellschaft für öffentliche Wirtschaft, hrsg. von Elisabeth Göbel u.a., Berlin, S. 48-60

Richter, Rudolf (1994), Institutionen ökonomisch analysiert, Tübingen

Riese, Christoph (1998), Vergaberecht - Grundlagen, Verfahren, Rechtsschutz, Berlin u.a.

Roggenkamp, Sybille (1999), Public Private Partnership - Entstehung und Funktionsweise kooperativer Arrangements zwischen öffentlichem Sektor und Privatwirtschaft, Frankfurt am Main u.a.

Rößl, Dietmar (1990), Die Entwicklung eines Bezugsrahmens und seine Stellung im Forschungsprozeß, in: Journal für Betriebswirtschaft, 40. Jg., Nr. 2, S. 99-110

Savas, Emanuel S. (2000), Privatization and Public-Private Partnership, New York und London

Schedler, Kuno und Isabella Proeller (2003), New Public Management, 2. Aufl., Bern, Stuttgart und Wien

Schinzer, Heiko (1999), Supply Chain Management, in: Wisu, 28. Jg., Nr. 6, S. 857-863

Schneider, Dieter (1983), Marketing als Wirtschaftswissenschaft oder Geburt einer Marketingwissenschaft aus dem Geiste des Unternehmensversagens?, in: Zeitschrift für betriebswirtschaftliche Forschung, 35. Jg., Nr. 3, S. 197-224

Schönsleben, Paul und Ralf Hieber (2002), Gestaltung von effizienten Wertschöpfungspartnerschaften im Supply Chain Management, in: Integriertes Supply Chain Management - Theorie und Praxis effektiver unternehmensübergreifender Geschäftsprozesse, hrsg. von Axel Busch und Wilhelm Dangelmaier, Wiesbaden, S. 45-62

Stölzle, W. (1999), Industrial Relationships, München, Wien und Oldenbourg

Schusser, Oliver (1999), Die Wertschöpfungskette als strategisches Planungsinstrument, in: Der Betriebswirt, 40. Jg., Nr. 2, 9-16

Schweitzer, Marcell (2000), Gegenstand und Methoden der Betriebswirtschaftslehre, in: Allgemeine Betriebswirtschaftslehre, Band 1, 8. Aufl., hrsg. von Franz X. Bea, Erwin Dichtl und Marcell Schweitzer, Stuttgart, S. 2379

Schweitzer, Marcell (2004), Gegenstand und Methoden der Betriebswirtschaftslehre, in: Allgemeine Betriebswirtschaftslehre, Band 1, 9. Aufl., hrsg. von Franz X. Bea, Birgit Friedl und Marcell Schweitzer, Stuttgart, S. 23-82

Specht, Dieter und Kai Hellmich (2000), Management der Zulieferbeziehungen in dynamischen Produktionsnetzen, in: Supply Chain Management, hrsg. von Horst Wildemann, München, S. 89-115

Tan, Keah C. (2001), A Framework of Supply Chain Management Literature, in: European Journal of Purchasing and Supply Management, 7. Jg., Nr. 3, S. 39-48

Thom, Norbert und Adrian Ritz (2000), Public Management - Innovative Konzepte zur Führung im öffentlichen Sektor, Wiesbaden

Thom, Norbert und Adrian Ritz (2003), Möglichkeiten der Wertschöpfungssteigerung durch Public Private Partnership, in: Geschäftsmodelle für Wertschöpfungsnetzwerke, hrsg. von Norbert Bach, Wolfgang Buchholz und Bernd Eichler, Wiesbaden, S. 435-457

von Bandemer, Stephan (2001), Qualitätsmanagement, in: Handbuch zur Verwaltungsreform, 2. Aufl., hrsg. von Bernhard Blanke u.a., Opladen, S. 414-424 
Wagner, Stephan M. (2001), Strategisches Lieferantenmanagement in Industrieunternehmen - Eine empirische Untersuchung von Gestaltungskonzepten, Frankfurt am Main u.a.

Werner, Hartmut (2000), Supply Chain Management - Partnerschaft zwischen Lieferant und Kunde, in: Wisu, 29. Jg., Nr. 6, S. 813-816

Weiber, Rolf und Jost Adler (1995), Informationsökonomisch begründete Typologisierung von Kaufprozessen, in: Zeitschrift für betriebswirtschaftliche Forschung, 47. Jg., Nr. 1, S. 43-65

Wildemann, Horst (2000), Von Just-in-Time zu Supply Chain Management, in: Supply Chain Management, hrsg. von Horst Wildemann, München, S. 49-85

Wildemann, Horst (2005), Wertorientierte Supply Chain Collaboration, in: Perspektiven des Supply Management Konzepte und Anwendungen, hrsg. von Michael Eßig, Berlin, Heidelberg und New York, S. 501-521

Williamson, Oliver E. (1990), Die ökonomischen Institutionen des Kapitalismus - Unternehmen, Märkte, Kooperationen, Tübingen

Williamson, Oliver E. (1991a), Comparative Economic Organization - The Analysis of Discrete Structural Alternatives, in: Administrative Science Quarterly, 36. Jg., Nr. 2, S. 269-296

Williamson, Oliver E. (1991b), Comparative Economic Organization - Vergleichende ökonomische Organisationstheorie - Die Analyse diskreter Strukturalternativen, in: Betriebswirtschaftslehre und ökonomische Theorie, hrsg. Dieter von Ordelheide, Dieter Rudolph, Bernd und Elke Büsselmann, Stuttgart, S. 13-49

Zafirowski, Milan (2001), Administration and Society - Beyond Public Choice?, in: Public Administration, 79. Jg., Nr. 3, S. 665-688

Zimmermann, Klaus (2003), Supply Chain Balanced Scorecard - Unternehmensübergreifendes Management von Wertschöpfungsketten, Wiesbaden 